\title{
Analysis of the Schwarz Waveform Relaxation Domain Decomposition Method for the Linear Schrödinger Equation
}

\author{
by \\ Moritz Hof \\ Submitted to the Faculty of Graduate Studies and Postdoctoral Affairs in \\ partial fulfillment of the requirements for the degree of \\ Master of Science in Applied Mathematics \\ at \\ Carleton University \\ Ottawa, Ontario \\ (C) 2016 \\ Moritz Hof
}




\title{
Analysis of the Schwarz Waveform Relaxation Domain Decomposition Method for the Linear Schrödinger Equation \\ by \\ Moritz Hof
}

\begin{abstract}
Submitted to the School of Mathematics and Statistics in partial fulfillment of the requirements for the degree of Master of Science in Applied Mathematics
\end{abstract}

\begin{abstract}
The goal of this thesis is to analyze the convergence and the rate of the convergence of the Classical and Optimal Schwarz Waveform Relaxation (CSWR and OSWR, respectively) domain decomposition methods for the Schrödinger equation. The analysis is derived from asymptotic symbolic expansions of inhomogeneous symbols of pseudo-differential operators associated to the linear Schrödinger equations.
\end{abstract}


Dedicated to Mareike Hedderich 


\section{Contents}

0.1 List of Symbols . . . . . . . . . . . . . . . . . vi

1 Introduction $\quad \mathbf{1}$

1.0.1 Notation and basics of distribution theory . . . . . . . . . . . . 2

1.0.2 Fourier Analysis on $\mathbb{R}^{n} \ldots \ldots \ldots$. . . . . . . . . . 4

1.0.3 Basics about pseudo-differential operators . . . . . . . . . . . . . . 5

1.0.4 Characteristics and Bicharacteristics . . . . . . . . . . . . . 10

1.0.5 Brief description of the Schwarz Waveform Relaxation Algorithm . . 14

2 Analysis of the SWR Algorithm 15

2.0.1 Well-Posedness of the algorithm . . . . . . . . . . . . 16

3 Convergence Rate of Schwarz Waveform Relaxation Algorithm 22

3.0.1 Asymptotic estimates of the contraction factor of the CSWR algorithm 25

3.0.2 Asymptotic estimates of the contraction factor in the OSWR algorithm 31

3.0.3 Convergence of the CSWR and OSWR algorithm . . . . . . . . . 37

4 Numerical Validation $\quad 39$

4.0.1 Numerical approximation .................... 39

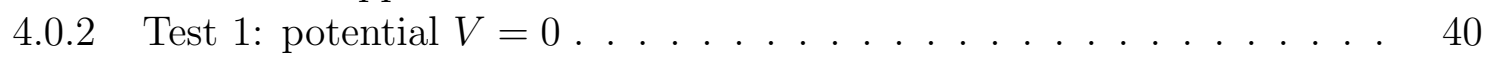

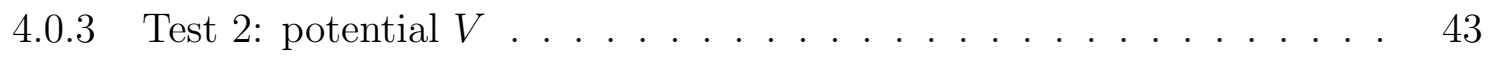

5 Conclusion $\quad 46$ 


\section{Acknowledgments}

I would like to take this opportunity to express my sincere gratitude to my supervisor, Emmanuel Lorin, whose extensive knowledge of the subject and interesting variety of research topics he proposed, kept me highly captivated. His sound advice and constant support not only enhanced my motivation and focus on the task, but also considerably helped to shape this work.

I would also extending my gratitude to the School of Mathematics and Statistics at Carleton University for accommodating me over the past two years.

Finally, I am truly grateful to my girlfriend for her everlasting belief in me and her continuous endorsement. 


\subsection{List of Symbols}

- $\mathcal{D}$ - the space of test functions

- $\mathcal{D}^{\prime}$ - the space of distributions

- $\mathcal{S}$ - the Schwartz space

- $\mathcal{F}$ - Fourier Transform 


\section{Chapter 1}

\section{Introduction}

The thesis is concerned with using a particular class of domain decomposition method (DDM) to solve the real-time linear Schrödinger equation (LSE). The methods involve two concepts: waveform relaxation algorithms and accurate transmission conditions [9]. This approach has led to efficient algorithms design for solving all kinds of the wave propagation problems.

Let us consider the following initial boundary-value problem: determine the complexvalued wavefunctions $u(x, t)$ solution to the LSE, set on $\mathbb{R}$,

$$
\begin{aligned}
& i \partial_{t} u=-\triangle u+V(x) u, x \in \mathbb{R}, t \geq 0, \\
& u(x, 0)=u_{0}(x), x \in \mathbb{R}
\end{aligned}
$$

where $u_{0}$ is the Cauchy data, $V$ is a real-valued space-dependent smooth potential, which is positive (respectively negative) for attractive (respectively repulsive) interactions. The usual system of equations in (1.1) has a factor of $(1 / 2)$ in front of the kinetic operator $-\Delta$, however it is omitted here since this thesis is dedicated to mathematical and numerical systems. We use a domain decomposition method, the Schwarz Waveform Relaxation (SWR) algorithm, to solve the initial value problem (1.1) by splitting it into two initial boundary value problems. Namely, in the one-dimensional case, the SWR algorithm splits the space $\mathbb{R}$ into two regions, with or without overlap. The SWR iterations allow for a reconstruction of the 
solution between the adjacent subdomains and exchange information on the boundary, called the interface. This in turn makes the problems on the subdomains independent, therefore making the domain decomposition methods suitable for parallel computing [6].

The goal of this thesis is to study the time dependent Schrödinger equation when using under the Schwarz Waveform Relaxation Method in one-dimension. In Section 2, we first introduce preliminary notations and then analyze the rate of convergence of both the CSWR and the OSWR with constant and general potential $V$. The analysis uses pseudo-differential operator theory associated with their asymptotic symbolic calculus to determine the convergence rates of the CSWR method. In Section 3, one proves the well-posedness of the SWR for the LSE, that is, the existence, uniqueness and the regularity of the solution. In Section 4, we numerically validate the theoretical convergence rates with the numerical convergence rates. We conclude in Section 5.

\subsubsection{Notation and basics of distribution theory}

Let $\Omega$ be an open subset of $\mathbb{R}^{n}$, and let $C^{k}(\Omega)$ where $k$ is a nonnegative integer, be the space of $k$-time differentiable functions on $\Omega$ in $\mathbb{C}$. If $k=\infty$, we speak of the space of indefinitely differentiable functions on $\Omega$ denoted $C^{\infty}(\Omega)$.

Muti-indices notation will be used to denote partial derivatives of functions in $C^{k}(\Omega)$. Let $\alpha=\left(\alpha_{1}, \ldots, \alpha_{n}\right)$ be an element of $\mathbb{N}^{n}$, then we define the modulus $|\alpha|=\left(\alpha_{1}+\cdots+\alpha_{n}\right)$ and $\alpha !=\alpha_{1} ! \cdots \alpha_{n} !$. It is useful to introduce the following notation $D_{j}=-i \frac{\partial}{\partial x_{j}}$ when dealing with the Fourier transformation. We shall denote higher order derivatives by $\partial^{\alpha}=\partial_{1}^{\alpha_{1}} \cdots \partial_{n}^{\alpha_{n}}$ or $D^{\alpha}=D^{\alpha_{1}} \ldots D^{\alpha_{n}}$. The convention of denoting monomials in the components of vectors in $\mathbb{R}^{n}$ is also used, that is, if $x \in \mathbb{R}^{n}$ to mean $x^{\alpha}=x_{1}^{\alpha_{1}} \cdots x_{n}^{\alpha_{n}}$. This notation is used throughout to describe differential operators.

Distributions are a class of linear functionals, which map a set of test functions into the set of real numbers. The set of test functions are described in $\mathcal{D}(\mathbb{R})$, which is the set of 
functions $\phi: \mathbb{R} \rightarrow \mathbb{R}$ having two properties: $\phi$ is infinitely differentiable, and $\phi$ has compact support, that is, $\phi$ is identically zero outside some closed and bounded interval $\Omega \subset \mathbb{R}$. By $\phi \in C_{0}^{\infty}$ we denote the space of functions in $C^{\infty}$ with compact support. We also write $\operatorname{supp} \phi$ for the compact support of $\phi$.

Definition 1.0.1. A distribution $T$ on an open set $\Omega$, is a linear form $u$ on $C_{0}^{\infty}(\Omega)$, which satisfies the following continuity property: there exists $m \in \mathbb{Z}$ and a constant $C$, such that for any compact subset $K$ of $\Omega$, we have that for any $\phi \in C_{0}^{\infty}(\Omega)$, which are zero outside of $K$,

$$
|T(\phi)| \leq C \sup _{x \in K} \sup _{|\alpha| \leq m}\left|\partial^{\alpha} \phi(x)\right|
$$

It is convention to usually describe a distribution $T$ as a linear mapping $T: \mathcal{D}(\mathbb{R}) \rightarrow \mathbb{R}$, where $T(\phi)$ is written $\langle T, \phi\rangle$ for the value of $T$ acting on a test function $\phi$.

The space of distributions on $\Omega$ is then the dual of $\mathcal{D}(\Omega)$ and is denoted $\mathcal{D}^{\prime}(\Omega)$.

Example: If $f: \mathbb{R} \rightarrow \mathbb{R}$ is a locally integrable function. Then a corresponding distribution $T_{f}$ is defined by

$$
\left\langle T_{f}, \phi\right\rangle=\int f(x) \phi(x) d x
$$

In particular, this integral depends linearly and continuously on $\phi$.

Definition 1.0.2. A differential operator on $\Omega$ is a finite linear combination of derivatives of arbitrary order with coefficients in $C^{\infty}(\Omega)$. The operator has order $m$, if the highest order of the derivatives is $m$.

$$
P=\sum_{|\alpha| \leq m} a_{\alpha}(x) D^{\alpha}
$$

where $a \in C^{\infty}(\Omega)$ are the coefficients of $P$. Notice that this defines a linear mapping between $C^{k+m}(\Omega)$ into $C^{k}(\Omega)$. Then the symbol $p$ of $P$, is the polynomial function in $(x, \xi)$ defined on $\Omega \times \mathbb{R}^{n}$ by

$$
p(x, \xi)=\sum_{|\alpha| \leq m} a_{\alpha}(x) \xi^{\alpha}
$$


where the principle symbol of order $m$ is the homogeneous function in $(x, \xi)$

$$
p(x, \xi)=\sum_{|\alpha|=m} a_{\alpha}(x) \xi^{\alpha}
$$

Let $p(\xi)$ be a polynomial in $n$ variables $\xi_{1}, \ldots, \xi_{n}$ with complex coefficients and let $P(D)$ be a differential operator. If $\xi_{j}$ is replaced by $D_{j}$, then this gives a one-to-one correspondence between polynomials and differential operator, which have constant coefficients:

$$
P(D) e^{i\langle x, \xi\rangle}=p(\xi) e^{i\langle x, \xi\rangle}
$$

where $\langle x, \xi\rangle=x_{1} \xi_{1} \ldots x_{n} \xi_{n}, n \in \mathbb{N}$.

\subsubsection{Fourier Analysis on $\mathbb{R}^{n}$}

Let us first introduce a particular space of interest, namely the Schwartz space $\mathcal{S}\left(\mathbb{R}^{n}\right)$. The space $\mathcal{S}\left(\mathbb{R}^{n}\right)$ is the space of $C^{\infty}$ functions $u$ on $\mathbb{R}^{n}$, which satisfy

$$
\forall \alpha \in \mathbb{N}^{n}, \forall \beta \in \mathbb{N}^{n}, \quad \sup _{x \in \mathbb{R}^{n}}\left|x^{\alpha} \partial^{\beta} u(x)\right|<\infty
$$

Example: If $a$ is a positive real number, then

$$
e^{-a x^{2}} \in \mathcal{S}(\mathbb{R})
$$

The space $\mathcal{S}\left(\mathbb{R}^{n}\right)$ is then described with the semi-norm defined as above with $\alpha$ and $\beta$ running over $\mathbb{N}^{n}$. If $x=\left(x_{1}, \ldots, x_{n}\right)$ is a vector in $\mathbb{R}^{n}$, we define the modulus or $\ell^{2}-$ norm to be

$$
|x|=\left(\sum_{j=1}^{n} x_{j}^{2}\right)^{1 / 2}
$$

The Schwartz space $\mathcal{S}\left(\mathbb{R}^{n}\right)$, is otherwise known as the space of rapidly decreasing functions.

Definition 1.0.3. The Fourier Transform $\mathcal{F}: \mathcal{S}\left(\mathbb{R}^{n}\right) \rightarrow \mathcal{S}\left(\mathbb{R}^{n}\right)$ acting on $u$, denoted $\hat{u}$, is 
defined

$$
\mathcal{F}(u)(\xi)=\hat{u}(\xi)=\int_{\mathbb{R}^{n}} e^{-i\langle x, \xi\rangle} u(x) d x, \text { for any } \xi \in \mathbb{R}^{n}
$$

where $\langle x, \xi\rangle=\sum_{j=1}^{n} x_{j} \xi_{j}$ is the inner product in $\mathbb{R}^{n}$.

The Fourier operator $\mathcal{F}$ has the following important properties for $u \in \mathcal{S}\left(\mathbb{R}^{n}\right)$ and $\xi \in \mathbb{R}^{n}$

$$
\begin{aligned}
\mathcal{F}\left(D_{j} u\right)(\xi) & =\xi_{j} \hat{u}(\xi), \\
\mathcal{F}\left(\tau_{y} u\right)(\xi) & =e^{i\langle y, \xi\rangle} \hat{u}(\xi),
\end{aligned}
$$

where $\tau_{y} u(x)=u(x+y)$.

$$
\begin{aligned}
\mathcal{F}\left(x_{j} u\right)(\xi) & =-D_{j} \hat{u}(\xi) \\
\mathcal{F}\left(e^{-i\langle x, \nu\rangle} u\right)(\xi) & =\tau_{\nu} \hat{u}(\xi) .
\end{aligned}
$$

Definition 1.0.4. A function $a \in C^{\infty}\left(\mathbb{R}^{n}\right)$ is said to be slowing increasing if

$$
\forall \alpha \in \mathbb{N}^{n}, \exists M_{\alpha} \in \mathbb{N}, \exists C_{\alpha}>0, \forall x \in \mathbb{R}^{n},\left|\partial^{\alpha} a(x)\right| \leq C_{\alpha}(1+|x|)^{M_{\alpha}}
$$

Any linear form $u$ on $\mathcal{S}\left(\mathbb{R}^{n}\right)$ that has the semi-norm, which is described above (1.2), is called a tempered distribution on $\mathbb{R}^{n}$. The space of these tempered distributions is denoted $\mathcal{S}^{\prime}\left(\mathbb{R}^{n}\right)$. Thus the tempered distributions are as characterized as slowly increasing, meaning that each derivative of $u$ grows at most as fast as some polynomial. This characterization is dual to the rapidly falling behaviour of the derivatives of a function in the Schwartz space. We need this definition to allow us to introduce the notion pseudo-differential operators.

\subsubsection{Basics about pseudo-differential operators}

In this section, we give a few definitions and some important results related to pseudodifferential operators. We will introduce the definition of a symbol associated to pseudo- 
differential operators, asymptotic sums of symbols, and classical pseudo-differential symbol.

Definition 1.0.5. Let $m \in \mathbb{R}$. Let $S^{m}=S^{m}\left(\mathbb{R}^{n} \times \mathbb{R}^{n}\right)$ denote the set of all $a \in C^{\infty}\left(\mathbb{R}^{n} \times \mathbb{R}^{n}\right)$, such that

$$
\forall \alpha \in \mathbb{N}^{n}, \forall \beta \in \mathbb{N}^{n},\left|\partial_{x}^{\alpha} \partial_{\xi}^{\beta} a(x, \xi)\right| \leq C_{\alpha, \beta}(1+|\xi|)^{m-|\beta|} .
$$

We will also denote $S^{-\infty}=\cap_{m} S^{m}$. If $a \in S^{m}$, then a is said to be a symbol of order $m$.

Later in the thesis one will introduce the notion of asymptotic sums used on pseudodifferential symbols, therefore some properties are introduced to make the discussion later in the paper more precise. Let us define a semi-norm on $S^{m}\left(\mathbb{R}^{n} \times \mathbb{R}^{n}\right)$ by

$$
|a|_{\alpha, \beta}^{m}=\sup _{(x, \xi) \in \mathbb{R} \times \mathbb{R}}\left\{(1+|\xi|)^{-(m-|\beta|)}\left|\partial_{x}^{\alpha} \partial_{\xi}^{\beta} a(x, \xi)\right|\right\}
$$

We thus obtain a complete space [1], where the convergence $a_{n} \rightarrow a$ as $n \rightarrow \infty$ means that $\forall \alpha, \forall \beta,\left|a_{n}-a\right|_{\alpha, \beta}^{m} \rightarrow 0$.

Lemma 1.0.1. Let $a \in S^{0}\left(\mathbb{R}^{n} \times \mathbb{R}^{n}\right)$ and set $a_{\epsilon}(x, \xi)=a(x, \epsilon \xi)$. Then $a_{\epsilon}$ is bounded in $S^{0}$, and $a_{\epsilon} \rightarrow a$ in $S^{m}$ for all $m>0$, where $\epsilon \rightarrow 0$.

Proof. See [1].

Let $a_{j} \in S^{m_{j}}\left(\mathbb{R}^{n} \times \mathbb{R}^{n}\right)$, then

$$
a \sim \sum_{j} a_{j}
$$

where the relation $\sim$ means an asymptotic sum as $|\xi| \rightarrow \infty$, i.e.

$$
\forall k \geq 0, \quad a-\sum_{j=0}^{k} a_{j} \in S^{m_{k+1}}\left(\mathbb{R}^{n} \times \mathbb{R}^{n}\right)
$$

Note that in practice, decreasing sequence $\left\{m_{j}\right\}_{j \in \mathbb{N}}$ is often taken as $m_{j}=m-j$ or $m_{j}=$ $m-j / 2, j \in \mathbb{N}$. We now present the following proposition [1], about the existence of an asymptotic expansion for an symbol $a$ in $S^{m}\left(\mathbb{R}^{n}\right), m \in \mathbb{N}$. 
Proposition 1.0.1. There exists $a \in S^{m_{0}}\left(\mathbb{R}^{n} \times \mathbb{R}^{n}\right)$ such that $a \sim \sum a_{j}$. Moreover, we have that $\operatorname{supp} a \subset \cup_{j} \operatorname{supp} a_{j}$.

We repeat the proof from [1].

Proof. We look at the asymptotics for $1 /|\xi| \rightarrow 0$. Thus we take

$$
a=\sum_{j} \tilde{a}_{j}=\sum_{j}\left(1-\chi\left(\epsilon_{j} \xi\right)\right) a_{j}
$$

where $\chi \in C_{0}^{\infty}, \chi=1$, and $\epsilon_{j} \rightarrow 0$ sufficiently rapidly. Since $1-\chi(\epsilon \xi)$ tends to 0 in $S^{1}$, then lemma (1.0.1) implies that

$$
\left|\partial_{x}^{\alpha} \partial_{\xi}^{\beta} \tilde{a}_{j}\right| \leq 2^{-j}(1+|\xi|)^{m_{k+1}-|\beta|}
$$

if $|\alpha|+|\beta| \leq j$. This implies that the sum is locally finite, and therefore $a \in C^{\infty}\left(\mathbb{R}^{n} \times \mathbb{R}^{n}\right)$ For $\alpha, \beta, k$ given, we have that $N \geq|\alpha|+|\beta|$ and $m_{N}+1 \leq m_{k+1}$,

$$
\left|\partial_{x}^{\alpha} \partial_{\xi}^{\beta}\left(a-\sum_{j \leq N-1} \tilde{a}_{j}\right)\right| \leq(1+|\xi|)^{m_{k+1}-|\beta|} ;
$$

Therefore, we have that

$$
a-\sum_{j \leq k} a_{j}=a-\sum_{j \leq N-1} \tilde{a}_{j}+\sum_{k+1 \leq j \leq N-1} \tilde{a}_{j}+\sum_{j \leq k} \tilde{a}_{j}-a_{j}
$$

satisfies

$$
\left|\partial_{x}^{\alpha} \partial_{\xi}^{\beta}\left(a-\sum_{j \leq k} \tilde{a}_{j}\right)\right| \leq C_{\alpha, \beta, k}(1+|\xi|)^{m_{k+1}-|\beta|}
$$

since $\tilde{a}_{j}-a_{j} \in S^{\infty}\left(\mathbb{R}^{n} \times \mathbb{R}^{n}\right)$ and $\tilde{a}_{j} \in S^{m_{j}}\left(\mathbb{R}^{n} \times \mathbb{R}^{n}\right)$

That is, there is a symbol in the space of rapidly decreasing functions such that the symbol a can be approximated by an asymptotic sum in $\mathcal{S}\left(\mathbb{R}^{n}\right)$. 
Definition 1.0.6. A symbol $a \in S^{m}\left(\mathbb{R}^{n} \times \mathbb{R}^{n}\right)$ is said to be a classical if $a \sim \sum_{j} a_{j}$, where the functions $a_{j}$ are homogeneous of degree $m-j$ for $|\xi| \geq 1$, that is, $a_{j}(x, \lambda \xi)=\lambda^{m-j} a_{j}(x, \xi)$ for $|\xi| \geq 1, \lambda \geq 1$.

We know present that following using fact given in [1].

Proposition 1.0.2. If $a \in S^{m}\left(\mathbb{R}^{n} \times \mathbb{R}^{n}\right)$ and $u \in \mathcal{S}\left(\mathbb{R}^{n}\right)$, and $x \in \mathbb{R}$, the following expression

$$
O p(a) u(x)=(2 \pi)^{-n} \int a(x, \xi) \hat{u}(\xi) e^{i x \xi} d \xi
$$

defines a operator on $\mathcal{S}\left(\mathbb{R}^{n}\right)$, and

$$
(a, u) \mapsto O p(a) u
$$

is continuous.

Proof. Since $\hat{u} \in \mathcal{S}\left(\mathbb{R}^{n}\right)$ and $a \in S^{m}\left(\mathbb{R}^{n} \times \mathbb{R}^{n}\right)$, we have that

$$
\begin{aligned}
|O p(a) u(x)| & =\left|(2 \pi)^{-n} \int a(x, \xi) \hat{u}(\xi) e^{i x \xi} d \xi\right| \\
& \leq(2 \pi)^{-n} \sup \left|a(x, \xi)(1+|\xi|)^{-m}\right| \int(1+|\xi|)^{-m}|\hat{u}(\xi)| d \xi
\end{aligned}
$$

Therefore $O p(a)$ is bounded and thus continuous.

The convergence rates for the classical and optimized Schwarz Waveform Relaxation algorithm for the one-dimensional real-time Schrödinger are computed by using symbolic asymptotic expansions associated to pseudo-differential operators. Therefore, we shall begin with an introduction to these operators in a relevant framework. 
Definition 1.0.7. A pseudo-differential operator $\mathcal{A}\left(x, t, \partial_{t}\right):=O p(a)$ is defined by

$$
\begin{aligned}
\mathcal{A}\left(x, t, \partial_{t}\right)(u(x, t)) & =\mathcal{F}_{t}^{-1}(a(x, t, \tau)(\hat{u}(x, \tau)) \\
& =\int_{\mathbb{R}} a(x, t, \tau) \mathcal{F}_{t}(x, t) e^{i t \tau} d \tau \\
& :=\operatorname{Op}(a) u(x, t)
\end{aligned}
$$

where $a(x, t, \tau)$ is the symbol the symbol of a, and where $\mathcal{F}_{t}$ in the Fourier transform in $t$

$$
\mathcal{F}_{t}(u)(x, \tau)=\hat{u}(x, \tau)=\frac{1}{2 \pi} \int_{\mathbb{R}} u(x, t) e^{-i t \tau} d \tau
$$

If we have a symbol $a \in S^{m}\left(\mathbb{R}^{n} \times \mathbb{R}^{n}\right)$, then one says a pseudo-differential operator $\mathcal{A}$ is an element of the class $O P S^{m}\left(\mathbb{R}^{n} \times \mathbb{R}^{n}\right)$.

Example: We consider $\partial_{t}$ and we take the Fourier transform in $t$ with co-variable $\tau$, we get the associated symbol $\tau \mapsto i \tau \in S^{1}\left(\mathbb{R}^{n} \times \mathbb{R}^{n}\right)$ :

$$
\begin{aligned}
\mathcal{F}_{t}\left(\partial_{t} u(x, t)\right) & =\frac{1}{2 \pi} \int_{\mathbb{R}} \partial_{t} u(x, t) e^{-i \tau t} d t \\
& =i \tau \frac{1}{2 \pi} \int_{\mathbb{R}} \partial_{t} u(x, t) e^{-i \tau t} d t \\
& =i \tau \mathcal{F}_{t}(u(x, t))
\end{aligned}
$$

We will be using inhomogeneous pseudo-differential operators, which were introduced in [11] and applied in various papers [2], [3], to determine transparent boundary conditions (TBCs) and artificial boundary conditions (ABCs), which will need to be used as a transmission condition in the SWR-framework. Since all details cannot be expressed here, we shall only introduce the important concepts.

Definition 1.0.8. Let $\alpha \in \mathbb{R}$ and an open set $\Xi$ of $\mathbb{R}$. The symbol class $\mathbb{S}^{\alpha}(\Xi \times \Xi)$ is defined to be a linear space of $\mathcal{C}^{\infty}$ symbol functions $a\left(x, t, \partial_{t}\right) \in \Xi \times \Xi \times \mathbb{R}$ such that for any set 
$K \subseteq \Xi \times \Xi$ for all indices $\beta, \delta, \gamma$, there exists a constant $C_{\beta, \delta, \gamma}>0$, such that

$$
(\forall(x, t) \in \mathrm{K})(\forall \tau \in \mathbb{R})\left|\partial_{\tau}^{\beta} \partial_{t}^{\delta} \partial_{x}^{\gamma} a(x, t, \tau)\right| \leq C_{\beta, \delta, \gamma}\left((K)\left(1-|\tau|^{2}\right)^{\alpha-\beta}\right)
$$

A function $f$ is said to be inhomogeneous of degree $m$ if: $f(x, t, \mu \tau)=\mu^{m} f(x, t, \tau)$, for any $\mu>0$. The definition leads us to the definition of an inhomogeneous operator. A pseudodifferential operator $\mathcal{A}=\mathcal{A}\left(x, t, \partial_{t}\right)$ is inhomogeneous of classical order $M$ for $M \in \mathbb{Z} / 2$, if its total symbol $a=\sigma(a)$ permits the following asymptotic expansion

$$
a(x, t, \tau) \sim \sum_{j=0}^{\infty} a_{M-j / 2}(x, t, \tau)
$$

where $a_{M-j / 2}$ is an inhomogeneous function of degree $M-j / 2$ for $j \in \mathbb{N}$, and where the relation $\sim$ is defined as

$$
\forall \widetilde{m} \in \mathbb{N}, \quad a-\sum_{j=0}^{\infty} a_{M-j / 2} \in S^{M-(\tilde{m}+1) / 2}
$$

We denote $a \in S_{S}^{\mathrm{M}}(\Xi \times \Xi)$, if $a$ is a symbol that satisfies the above expansion and its associated pseudo-differential operator $\mathcal{A}=\mathrm{Op}(a)$ is an element in $\operatorname{OPS}_{\mathrm{S}}^{\mathrm{M}}(\Xi \times \Xi)$.

\subsubsection{Characteristics and Bicharacteristics}

This section recalls some properties of characteristics and bicharacteristics presented in [10]. Recall that a differential operator $P$ on an open set $\Omega \subset \mathbb{R}^{n}$ is a linear mapping

$$
u \mapsto P u=\sum_{\alpha} a^{\alpha} D^{\alpha} u
$$

where $a^{\alpha}$ is a function, which is defined in $\Omega$ and that all but a finite number of coefficients of $a^{\alpha}$ vanish identically on any compact set of $\Omega$, that is $\operatorname{supp} a^{\alpha}$ is locally finite. If $a^{\alpha} \in C^{k}(\Omega)$ for every $\alpha$, then $P$ has $C^{k}$ coefficients, therefore we have that $P$ is a linear mapping from 
$C^{\infty}(\Omega)$ into $C^{k}(\Omega)$. Let $P$ be a differential operator in $\Omega \subset \mathbb{R}^{n}$, the order $m$ of $P$ in $\Omega$ is the least upper bound of $|\alpha|$ for all $\alpha$ such that the coefficient $a^{\alpha} \neq 0$. If $P$ is a differential operator of finite order $m$, the principle part of $P$ is defined to be a polynomial in $\xi$ such that

$$
P_{m}(\xi)=P_{m}(x, \xi)=\sum_{|\alpha|=m} a^{\alpha} \xi^{\alpha}
$$

where $x \in \Omega$ and $\xi \in \mathbb{C}^{n}$. In order to pass to a manifold $\Omega$, an alternative expression is given. For some $\phi \in C^{\infty}(\Omega)$ and if $\tau$ is a parameter, it follows that

$$
e^{-i \tau \phi} P e^{i \tau \phi}=\tau^{m} P_{m}(\nabla \phi)+\ldots
$$

where the dots represent a polynomial of degree less than $m$ with respect to $\tau$. For a suitable choice of $\phi$, we are lead to the following definition, if $\Omega$ is a manifold and $P$ is a differential operator with continuous coefficients.

Definition 1.0.9. A surface $\phi(x)=\phi\left(x^{0}\right)$ where $\phi \in C^{1}(\Omega)$ and $\nabla \phi\left(x^{0}\right) \neq 0$ is said to be characteristic at $x^{0}$ with respect to the differential operator $P$ of order $m$ if $P_{m}(\nabla \phi)=0$ at $x^{0}$. If one can find $\psi$ such that $P_{m}(\nabla(\phi+\epsilon \psi))$ is not $O\left(\epsilon^{2}\right)$ at $x^{0}$ when $\epsilon \rightarrow 0$, the surface is the called a simply characteristic.

We will now introduce the classical method [10] of integration for the characteristic equation $P_{m}(x, \nabla \phi)=0$. Assume that $P_{m}$ has real $C^{2}$ coefficients in an open set $\Omega \subset \mathbb{R}^{n}$ and that $\phi \in C^{2}(\Omega)$ is a real valued function that has surfaces which are simply characteristics everywhere in $\Omega$. After differentiating the equation $P_{m}(x, \nabla \phi)=0$, we obtain

$$
\sum_{k=1}^{n} P_{m}^{(k)}(x, \nabla \phi) \partial^{2} \phi / \partial x_{j} \partial x_{k}+P_{m, j}(x, \nabla \phi)=0
$$

where

$$
P_{m}^{(j)}(x, \xi)=\partial_{\xi_{j}} P_{m}(x, \xi) ; \quad P_{m, j}(x, \xi)=\partial x_{j} P_{m}(x, \xi)
$$


Since $P_{m}^{(k)}(x, \nabla \phi)$ is continuously differentiable and

$$
\sum_{k=1}^{n}\left|P_{m}^{(k)}(x, \nabla \phi)\right|^{2} \neq 0
$$

everywhere in $\Omega$, since $\phi$ is a simple characteristic, the system of ordinary differential equations

$$
d x_{k} / d t=P_{m}^{(k)}(x, \nabla \phi), \quad k=1, \ldots, n
$$

defines a unique curve through every point in $\Omega$ without singularities and it follows from (1.11) that on this curve

$$
\frac{d}{d t} \partial \phi / \partial x_{j}=-P_{m, j}(x, \nabla \phi)
$$

If we let $\xi=\nabla \phi$ and consider it as a function of $t$ on the curve (1.13), we obtain the following Hamilton equations

$$
d x_{k} / d t=P_{m}^{(k)}(x, \xi), \quad d \xi_{k} / d t=-P_{m, k}(x, \xi), \quad k=1, \ldots, n
$$

Thus by Euler's Identity for homogeneous polynomials and along the curve defined by (1.13), we get

$$
d \phi / d t=\sum_{k=1}^{n} \frac{\partial \phi}{\partial x_{k}} \frac{d x_{k}}{d t}=m P_{m}(x, \nabla \phi)=0 .
$$

Therefore, $\phi$ is constant along the curve (1.12).

Definition 1.0.10. A solution to the Hamiltonian equations (1.14) for $P_{m}(x, \xi)=0$ but $P_{m}^{(k)}(x, \xi) \neq 0$ for some $k$ is called be a bicharacteristic strip, and the curve described by $x$ is called a bicharacteristic curve.

The above definition implies that it is sufficient to assume that $P_{m}(x, \xi)=0$ at one point of the strip because then it follows that $P_{m}(x, \xi)=0$ along the entire strip. In geometric terms, a bicharacteristic strip is a curve with a tangent plane with normal $\xi$ given at every point. The derivation of the Hamilton equations demonstrates that if $\phi$ is a solution to the characteristic equation $P_{m}(x, \nabla \phi)=0$, and also we define the bicharacteristic strip with the 
initial data $x=x^{0}$ and $\xi=\nabla \phi\left(x^{0}\right)$, then we get that $\xi=\nabla \phi(x)$ and $\phi=$ constant on the entire strip.

The notion of bicharacteristic strips are of importance since they allow us to derive the Transparent Boundary Conditions and Artificial Boundary Conditons (TBC/ABC) for wave equations [3]. For $P \in O P S^{m}$, the leading symbol $P_{m}(x, \xi)$, has a degree of homogeneity equal to $m$ and the bicharacteristic strips are integral curves of the Hamilton equations (1.14). After solving the Hamilton equations, we can see that the solutions propagate along the bicharacteristics strips. If $P_{m}\left(x_{0}, \xi_{0}\right)=0$, such strips are called the null-bicharacteristic strips. The union of all the null-bicharacteristics is called the wavefront $W F(u)$, which emanate from the points where the principle symbol is 0 . When determining the ABCs, one must identify the point $\left(x_{0}, \xi_{0}\right)$, which belongs to the wavefront. Next, we differentiate between the ingoing and outgoing bicharacteristics strips at the boundary. The operator $P$ can be factorized micro-locally into pseudo-differential operators, which correspond to outgoing or incoming strips. The factorization of $\mathrm{P}$ is due to the following theorem [1]:

Theorem 1.0.1. Let $P$ be a differential operator of the form

$$
P=\sum_{k+l \leq m} a_{k, l} D_{x}^{k} D_{t}^{l} \quad\left(a_{0, m} \equiv 0\right) .
$$

Then $P$ can be factorized into the form

$$
P\left(x, t, D_{x}, D_{t}\right)=\left(D_{t}-\lambda_{1}(t, x) D_{x}\right) \ldots\left(D_{t}-\lambda_{1}(t, x) D_{x}\right)+\text { order } m-1 \text { terms }
$$

where the $\lambda_{j} \xi$ are the distinct roots of the polynomial $p_{m}=\sum_{k+k=m} a_{k, l} \xi^{k} \tau^{l}=0$. Here: $D_{x, t}:=i \partial_{x, t}$. 


\subsubsection{Brief description of the Schwarz Waveform Relaxation Al- gorithm}

In this section, we give a brief description of the Classical (respectively Optimal) Schwarz Waveform Relaxation Algorithm (CSWR) (respectively (OSWR)). These algorithms allow one to solve space-dependent partial differential equation in subdomains "in parallel".

Suppose we would like to solve an equation $\mathcal{P}\left(t, x, \partial_{t}, \partial_{x}\right) \phi=f$ in the spatial domain $\Omega$ and time domain $(0, T)$, with initial data $\phi_{0}$. In its simplest form, the Schwarz waveform relaxation consists of splitting the domain $\Omega$ into subdomains $\Omega_{\epsilon}^{ \pm}$, with $\left(\Omega_{\epsilon}^{+} \cap \Omega_{\epsilon}^{-} \neq \emptyset\right)$ or without overlap $\left(\Omega_{\epsilon}^{+} \cap \Omega_{\epsilon}^{-}=\emptyset\right)$. The CSWR algorithm consists on solving subproblems in $\Omega_{\epsilon}^{+} \times(0, T)$ and $\Omega_{\epsilon}^{-} \times(0, T)$ iteratively, using boundary conditions, called transmission conditions at the interfaces $\Gamma_{\epsilon}^{+}=\{\epsilon / 2\}$ and $\Gamma_{\epsilon}^{-}=\{-\epsilon / 2\}$, where the values are calculated from the previous iteration in the adjacent subdomain. For $k \geq 1$,

$$
\begin{cases}P \phi^{ \pm,(k)} & =f, \text { in } \Omega_{\epsilon}^{ \pm} \times(0, T), \\ \phi^{ \pm,(k)}(\cdot, 0) & =\phi_{0}, \text { in } \Omega_{\epsilon}^{ \pm}, \\ \phi^{ \pm,(k)}( \pm \epsilon, \cdot) & =\phi^{ \pm,(k-1)}(\mp \epsilon / 2, \cdot), \text { on } \Gamma_{\epsilon}^{ \pm} \times(0, T)\end{cases}
$$

with a given initial guess $\phi^{ \pm,(0)}( \pm \epsilon, \cdot)$. The Optimized Schwarz Waveform Relaxation algorithms is described in the same manner as the CSWR algorithm except that we use a transparent transmission conditions between the subdomains [3]. The algorithm then consists of introducing operators $\mathcal{B}_{ \pm}$, usually pseudo-differential operators, and $k \geq 1$, we have

$$
\left\{\begin{array}{l}
P \phi^{ \pm,(k)}=f, \text { in } \Omega_{\epsilon}^{ \pm} \times(0, T), \\
\phi^{ \pm,(k)}(\cdot, 0)=\phi_{0}, \text { in } \Omega_{\epsilon}^{ \pm}, \\
\mathcal{B}_{ \pm}^{(k)} \phi^{ \pm,(k)}=\mathcal{B}_{ \pm}^{(k-1)} \phi^{\mp,(k-1)}, \text { on } \Gamma_{\epsilon}^{ \pm} \times(0, T)
\end{array}\right.
$$

with given initial guess $\phi^{ \pm,(0)}( \pm \epsilon, \cdot)$. 


\section{Chapter 2}

\section{Analysis of the SWR Algorithm}

Let us consider the following initial boundary-value problem: determine the complex-valued wavefunctions $u(x, t)$ solution to the real-time linear Schrödinger equation set on $\mathbb{R}$,

$$
\left\{\begin{array}{l}
i \partial_{t} u=-\triangle u+V(x) u, x \in \mathbb{R}, t \geq 0 \\
u(x, 0)=u_{0}(x), x \in \mathbb{R}
\end{array}\right.
$$

as described in (1.1). We then can define the real time-dependent one-dimensional Schrödinger operator $P$ as

$$
P\left(x, t, \partial_{x}, \partial_{t}\right)=i \partial_{t}+\partial_{x}^{2}-V(x)
$$

The Schwarz waveform relaxation algorithm associated with (1.1) is as follows: First one decomposes the spatial domain $\Omega=\mathbb{R}$ into overlapping subdomains $\Omega_{\epsilon}^{ \pm}$with $\Omega_{\epsilon}^{+}=(-\infty, \epsilon / 2)$ and $\Omega_{\epsilon}^{-}=(-\epsilon / 2, \infty)$ with $\epsilon>0$. The Schwarz waveform relaxation with overlap corresponds to iteratively solving subproblems in $\Omega_{\epsilon}^{+} \times(0, T)$ and $\Omega_{\epsilon}^{-} \times(0, T)$ using the transmission conditions at the interfaces $x=-\epsilon / 2$ and $x=\epsilon / 2$, where the values are attained from the 
previous Schwarz iteration. The algorithm for the iteration index $k=1,2, \ldots$ is given by

$$
\begin{cases}P \phi^{ \pm,(k)} & =0, \text { in } \Omega_{\epsilon}^{ \pm} \times \mathbb{R}_{+}^{*}, \\ \phi^{ \pm,(k)}(\cdot, 0) & =\phi_{0}^{ \pm}, \text {in } \Omega_{\epsilon}^{ \pm}, \\ \phi^{ \pm,(k)}( \pm \epsilon / 2, \cdot) & =\phi^{\mp,(k-1)}( \pm \epsilon / 2, \cdot), \text { in } \mathbb{R}_{+}^{*}\end{cases}
$$

where $\phi_{0}^{ \pm}$denotes the restriction of $\phi_{0 \mid \Omega_{\epsilon}^{ \pm}}$to $\Omega_{\epsilon}^{ \pm}$, with $\Omega_{\epsilon}^{+}=(-\infty, \epsilon / 2), \Omega_{\epsilon}^{-}=(-\epsilon / 2,+\infty)$ and $\varepsilon>0$ is the (small) size of the overlapping region. Working with the error equations, i.e. $e_{P}^{C, \pm}:=\phi_{0 \mid \Omega_{\epsilon}^{ \pm}}-\phi^{ \pm}$the CSWR in $\Omega_{\epsilon}^{ \pm}$, reads

$$
P \cdot e_{P}^{C, \pm}=0 \text { in } \Omega_{\epsilon}^{ \pm} \times \mathbb{R}_{+}^{*}, \quad e_{P}^{C, \pm}( \pm \epsilon / 2, t)=h_{\epsilon}^{ \pm}(t) \text { at }\{ \pm \epsilon / 2\} \times \mathbb{R}_{+}^{*},
$$

where $P$ is given by (2.1). The index $P$ in $e_{P}^{C, \pm}$ is introduced to specify the operators associated error, and the exponent $C$ stands for the CSWR algorithm (we will later use the index $O$ for the OSWR algorithm). We will also use other approximate errors when the potential $V$ is variable.

\subsubsection{Well-Posedness of the algorithm}

We study the well-posedness of the CSWR algorithm, and without loss of generality, we restrict ourselves to the subdomain problem in $\Omega_{\epsilon}^{+}$, that is

$$
\left\{\begin{array}{l}
P \phi=f, \text { in } \Omega_{\epsilon}^{+} \times \mathbb{R}_{+}^{*} \\
\phi(\cdot, 0)=\phi_{0}, \text { in } \Omega_{\epsilon}^{+} \\
\phi(\epsilon / 2, \cdot)=h, \text { in } \mathbb{R}_{+}^{*}
\end{array}\right.
$$

The algorithm will then be well-defined if the initial boundary value problem for each subdomain is well-posed. The following proposition provides the existence, uniqueness and the regularity of the solution.

Proposition 2.0.1. Let the real space-dependent potential $V$ be in $L^{\infty}\left(\Omega_{\epsilon}^{+}\right)$. Suppose $f$ is 
in $H^{1}\left(0, T ; L^{2}\left(\Omega_{\epsilon}^{+}\right)\right), \phi_{0}$ in $H^{2}\left(\Omega_{\epsilon}^{+}\right), h$ in $H^{1}(0, T)$, with the compatibility conditions

$$
\phi_{0}(\epsilon / 2)=h(0)
$$

Then (2.4) has a unique solution $\phi$ in $H^{2,1}\left(\Omega_{\epsilon}^{+} \times(0, T)\right)$. Furthermore, suppose that $V$ is constant, $f$ in $H^{2}\left((0, T) \times \Omega_{\epsilon}^{+}\right), \phi_{0}$ in $H^{4}\left(\Omega_{\epsilon}^{+}\right)$. Then $\phi(-\epsilon / 2, \cdot)$ and $\partial_{x} \phi(-\epsilon / 2, \cdot)$ are in $H^{1}(0, T)$, and the following compatibility relation is satisfied:

$$
\lim _{t \rightarrow 0^{+}} \phi(\epsilon / 2, t)=\phi_{0}(-\epsilon / 2)
$$

Recall that $H^{r, s}(\Omega \times(0, T)):=L^{2}\left(0, T ; H^{r}(\Omega)\right) \cap H^{2}\left(0, T ; L^{2}(\Omega)\right)$ is an anisotropic Sobolev space.

Proof. We follow the analysis considered in [9].

(i) First a priori estimates. Multiplying equation (2.4) by $\bar{\phi}$, integrating by part in space, using the boundary condition and taking the imaginary parts, we get

$$
\frac{1}{2} \frac{d}{d t}\|\phi(\cdot, t)\|^{2}=|\phi(\epsilon / 2, t)|^{2}+\mathfrak{I m}(f(\cdot, t), \bar{\phi}(\cdot, t))-\bar{h} \partial_{x} \phi(\epsilon / 2, t)
$$

Here, we have used the fact that the potential $V$ is real. Now using Cauchy-Schwarz and the inequality

$$
\alpha \beta \leq \frac{\nu}{2} \alpha^{2}+\frac{1}{2 \nu} \beta^{2}, \text { for all } \alpha, \beta \in \mathbb{R}, \text { and } \nu>0
$$

applied to (2.6) and integrating with respect to time, we obtain

$$
\|\phi(\cdot, t)\|^{2} \leq\left|\phi_{0}\right|^{2}+\int_{0}^{t}\|f(\cdot, s)\|^{2} d s+\int_{0}^{t}|h(s)|^{2} d s+\int_{0}^{t}\|\phi(\cdot, s)\|^{2} d s+\int_{0}^{t}\left\|\partial_{x} \phi(\cdot, s)\right\|^{2} d s
$$

and taking the real part, we also have a bound in $H^{1}(0, T)$ for the last expression in the above relation: 


$$
\begin{aligned}
\int_{0}^{t}\left\|\partial_{x} \phi(\cdot, s)\right\|^{2} d s & =\mathfrak{R e}(f(\cdot, t), \bar{\phi}(\cdot, t)) \\
& \leq \frac{1}{2} \int_{0}^{t}\|f(\cdot, s)\|^{2} d s+\frac{1}{2} \int_{0}^{t}\|\phi(\cdot, t)\|^{2} d s
\end{aligned}
$$

Now are able to apply Grönwall's lemma, and we get

$$
\|\phi\|_{L^{\infty}\left((0, T) ; \Omega_{\epsilon}^{+}\right)}^{2} \leq e^{T}\left(\left\|\phi_{0}\right\|^{2}+\|f\|_{\left.L^{2}(0, T) ; \Omega_{\epsilon}^{+}\right)}^{2}+\|\phi(\epsilon / 2, \cdot)\|_{L^{2}(0, T)}^{2}+\|h\|_{L^{2}(0, T)}^{2}\right)
$$

We now apply (2.9) to $\partial_{t} \phi$ with the initial condition $\partial_{t} \phi(\cdot, t)=-i\left(f(\cdot, t)-\partial_{x x} \phi_{0}+V \phi_{0}\right)$ in $L^{2}\left(\Omega_{\epsilon}^{+}\right)$, the regularity assumptions on the data, and the Trace theorem [12] in time for $f$, we get

$$
\begin{aligned}
\left\|\partial_{t} \phi\right\|_{L^{\infty}\left(\Omega_{\epsilon}^{+}\right)}^{2}+ & \left\|\partial_{t} \phi(\epsilon / 2, \cdot)\right\|_{L^{2}\left(\Omega_{\epsilon}^{+}\right)}^{2} \leq \\
& C e^{T}\left(\left\|\phi_{0}\right\|_{H^{2}\left(\Omega_{\epsilon}^{+}\right)}^{2}+\|V\|_{L^{\infty}\left(\Omega_{\epsilon}^{+}\right)}^{2}\left\|\phi_{0}\right\|_{L^{2}\left(\Omega_{\epsilon}^{+}\right)}^{2}+\|f\|_{H^{1}\left((0, T) ; L^{\infty}\left(\Omega_{\epsilon}^{+}\right)\right)}^{2}+\|h\|_{H^{1}(0, T)}^{2}\right)
\end{aligned}
$$

(ii) Second a priori estimates. We apply the same analysis as we did above but we multiply (2.4) by $\partial_{t} \bar{\phi}$ and taking the real part instead of the imaginary part. We obtain

$$
-\frac{d}{d t}\left\|\partial_{x} \phi\right\|^{2}=-2 \mathfrak{R e}\left(h \partial_{t} \bar{\phi}(L, \cdot)\right)+2 \mathfrak{R e}\left(V \phi(\cdot, t), \partial_{t} \bar{\phi}(\cdot, t)+2 \mathfrak{R e}\left(f(\cdot, t), \partial_{t} \bar{\phi}(\cdot, t)\right)\right.
$$

which implies that we have

$$
\begin{gathered}
\left\|\partial_{x} \phi(\cdot, t)\right\|^{2} \leq\|\phi(\epsilon / 2, \cdot)\|_{L^{2}\left(0, T ; \Omega_{\epsilon}^{+}\right)}^{2}+2\left\|\partial_{t} \phi\right\|_{L^{2}\left((0, T) \times \Omega_{\epsilon}^{+}\right)}^{2} \\
+\|V\|_{L^{\infty}\left(\Omega_{\epsilon}^{+}\right)}\left\|\phi_{0}\right\|_{L^{2}\left((0, T) \times \Omega_{\epsilon}^{+}\right)}^{2}+\|f\|_{L^{2}\left((0, T) \times \Omega_{\epsilon}^{+}\right)}^{2} \\
+\|h\|_{L^{2}(0, T)}^{2}+\left\|\partial_{x} \phi_{0}\right\|_{H^{1}(0, T)}^{2}
\end{gathered}
$$

Then by (2.9) and (2.12), we deduce that

$$
\begin{aligned}
\left\|\partial_{x} \phi\right\|_{L^{\infty}\left((0, T) ; \Omega_{\epsilon}^{+}\right)}^{2} \leq & C e^{T}\left(\left\|\phi_{0}\right\|_{H^{1}\left(\Omega_{\epsilon}^{+}\right)}^{2}+\|V\|_{L^{\infty}\left(\Omega_{\epsilon}^{+}\right)}^{2}\left\|\phi_{0}\right\|_{L^{2}\left(\Omega_{\epsilon}^{+}\right)}^{2}\right. \\
& \left.+\|f\|_{H^{1}\left((0, T) ; L^{\infty}\left(\Omega_{\epsilon}^{+}\right)\right)}^{2}+\|h\|_{H^{1}(0, T)}^{2}\right)
\end{aligned}
$$


Finally, using $P \phi^{+}=f$ and (2.5), we have

$$
\begin{aligned}
\left\|\partial_{x x} \phi\right\|_{L^{\infty}\left((0, T) ; \Omega_{\epsilon}^{+}\right)}^{2} & \leq C e^{T}\left(\left\|\phi_{0}\right\|_{H^{1}\left(\Omega_{\epsilon}^{+}\right)}^{2}+\|V\|_{L^{\infty}\left(\Omega_{\epsilon}^{+}\right)}^{2}\left\|\phi_{0}\right\|_{L^{2}\left(\Omega_{\epsilon}^{+}\right)}^{2}\right. \\
& \left.+\|f\|_{H^{1}\left((0, T) ; L^{\infty}\left(\Omega_{\epsilon}^{+}\right)\right)}^{2}+\|h\|_{H^{1}(0, T)}^{2}\right)
\end{aligned}
$$

by $(2.9)-(2.13)$, we get a bound on $\phi$ in $H^{2,1}\left(\Omega_{\epsilon}^{+} \times(0, T)\right)$ and on $\phi(\epsilon / 2, \cdot)$ on $H^{1}(0, T)$. By Galerkin's method [12, 13], this is sufficient to obtain existence and uniqueness in those spaces. Moreover, we have that $\phi(\epsilon / 2, \cdot)$ in $H^{3 / 4}(0, T)$ by the Trace theorem [12] in $H^{2,1}\left(\Omega_{\epsilon}^{+} \times(0, T)\right)$.

(iii) Third a apriori estimate. We will now show that $\phi(\epsilon / 2, \cdot)$ is actually in $H^{1}(0, T)$ when $\epsilon>0$, and in order to simplify, we assume that the potential $V$ is constant. We may also assume that the solution $\phi$ of the Schrödinger equation $(1.1)$ is in $H^{4,2}\left(\Omega_{\epsilon}^{+} \times(0, T)\right)$ by [12]. As done in [9], we introduce the auxiliary problem satisfied by $z^{+}=e^{-t}\left(\phi^{+}-\phi\right)$ in $\Omega_{\epsilon}^{+} \times(0, T)$, which means:

$$
\left\{\begin{array}{l}
i \partial_{t} z+i z+\partial_{x x} z+V z=0 \text { in } \Omega_{\epsilon}^{+} \times(0, T) \\
z(\cdot, 0)=0, \text { in } \Omega_{\epsilon}^{+}, \\
z(\epsilon / 2, \cdot)=g, \text { in } \mathbb{R}_{+}^{*}
\end{array}\right.
$$

where $g(t)=e^{-t}(h(t)-\phi(\epsilon / 2, t))$ The boundary data $g$ is in $H^{1}(0, T)$. We are able to extend $g$ in $H^{1}(\mathbb{R})$ by $G$, vanishing in negative $t$, because of the compatibility condition (2.5). By the Fourier Transform in time with respect to $\tau$, we get a solution for (2.14):

$$
(i-\tau-V) \hat{z}+\partial_{x x} \hat{z}=0
$$

where we can obtain

$$
\hat{z}(x, \tau)=\hat{G}(\tau) e^{-2 \epsilon(\tau+V-i)^{1 / 2}}
$$


where $(\tau+V-i)^{1 / 2}$ has a negative imaginary part:

$$
(\tau+V-i)^{1 / 2}=\sqrt{\frac{\tau+V+\sqrt{(\tau+V)^{2}+1}}{2}}-i \sqrt{\frac{-\tau+V+\sqrt{(\tau+V)^{2}+1}}{2}}
$$

and $|\hat{z}(0, \tau) \leq| \hat{G}(\tau) \mid$, we have that

$$
\|z(\epsilon / 2, \cdot)\|_{H^{1}(0, T)} \leq\|g\|_{H^{1}(0, T)}
$$

which proves that $\phi(\epsilon / 2, \cdot)$ is in $H^{1}(0, T)$, and that

$$
\|\phi(\epsilon / 2, \cdot)\|_{H^{1}(0, T)}^{2} \leq C e^{T}\left(\|\phi\|_{H^{4,2}\left(\Omega_{\epsilon}^{+} \times(0, T)\right.}^{2}+\|h\|_{H^{1}(0, T}^{2}\right)
$$

To conclude that proof, we need to still prove 2.6. Since we have that $\phi$ is in $H^{4,2}\left(\Omega_{\epsilon}^{+} \times(0, T)\right)$, it satisfies

$$
\lim _{t \rightarrow 0^{+}} \phi(\epsilon / 2, t)=\phi_{0}(-\epsilon / 2)
$$

Therefore, we have to show that

$$
\lim _{t \rightarrow 0^{+}} z(\epsilon / 2, t)=0
$$

Since $G$ is supported in $\mathbb{R}_{+}, \hat{G}$ is analytic in the half-plane $\mathfrak{I m}<0$, and by $(2.15)$ and Paley-Wiener Theorem [14], we have that $z(\epsilon / 2, \cdot)$ is also supported in $\mathbb{R}_{+}$. Since we have just shown that $z(\epsilon / 2, \cdot)$ is in $H^{1}(0, T)$, and by the Sobolev Embedding theorem [7], we have that $\lim _{t \rightarrow 0_{+}} z(\epsilon / 2, t)=0$.

Theorem 2.0.1. Let $\epsilon>0$ and let $V$ in $L^{\infty}(\mathbb{R})$ and let $h_{+}$and $h_{-}$be given in $H^{1}(0, T)$. Then algorithm (2.4) defines a sequence of iterates $\left(\phi^{+,(k)}, \phi^{-,(k)}\right)$ in $H^{2,1}\left(\Omega_{\epsilon / 2}^{+} \times(0, T)\right) \times$ $H^{2,1}\left(\Omega_{\epsilon}^{-} \times(0, T)\right)$ with $\phi^{+,(k)}(-\epsilon / 2, \cdot), \partial_{x} \phi^{+,(k)}(-\epsilon / 2, \cdot), \phi^{-,(k)}(\epsilon / 2, \cdot)$ and $\partial_{x} \phi^{-,(k)}(\epsilon / 2, \cdot)$ in $H^{1}(0, T)$

Proof. The proof follows by induction using the previous Proposition (2.0.1) [9]. 
In the following chapter we discuss the the convergence rates for the CSWR and the OSWR algorithms, and present the main theorems about the contraction factors, which correspond to convergence rates for the two algorithms. 


\section{Chapter 3}

\section{Convergence Rate of Schwarz Waveform Relaxation Algorithm}

Considering again the Schrödinger operator $P(2.1)$, we now compute the theoretical convergence rates. We have the following proposition [1], which allows us to factorize the operator $P$.

Proposition 3.0.1. The Nirenberg-like factorization

$$
P\left(x, t, \partial_{x}, \partial_{t}\right)=\left(\partial_{x}+i \Lambda^{-}\right)\left(\partial_{x}+i \Lambda^{+}\right)+\mathcal{R}
$$

holds, where $\mathcal{R} \in \mathrm{OPS}^{-\infty}=\bigcap_{m} \mathrm{OPS}^{m}$ is a smooth pseudo-differential operator. The operators $\Lambda^{ \pm}$are pseudo-differential operators of order $1 / 2$ in time and order 0 in space. We also have that their total symbols $\lambda^{ \pm}$can be expanded in $S^{1 / 2}$ as

$$
\lambda^{ \pm} \sim \sum_{j=0}^{\infty} \lambda_{1 / 2-j / 2}
$$

where $\lambda_{1 / 2-j / 2}$ are elementary symbols corresponding to operators of order $1 / 2-j / 2, j \in \mathbb{N}$.

We can approximate $\lambda^{ \pm}$by computing a finite number of inhomogeneous symbols. 
Proposition 3.0.2. Let us fix the principal symbol to

$$
\lambda_{1 / 2}^{ \pm}=\mp \sqrt{-\tau+V(x)}
$$

Then, we have the following elementary symbols are given by

$$
\lambda_{0}^{ \pm}=0, \lambda_{-1 / 2}^{ \pm}=0 \text { and } \lambda_{-1}^{ \pm}=\mp \frac{i}{4} \frac{\partial_{x} V(x)}{-\tau+V(x)}
$$

The above propositions are proved [3] by using the following recursive formulae by fixing $\lambda_{1 / 2}^{+}$from the expression in (3.0.2). Then, for $j \in \mathbb{N}^{*}$,

$$
\lambda_{-j / 2}=\frac{1}{2 \lambda_{1 / 2}^{+}}\left(-i \partial_{x} \lambda_{1 / 2-j / 2}^{+}-\sum_{k=1}^{j} \lambda_{-j / 2+k / 2} \lambda_{1 / 2-k / 2}\right)
$$

Proposition 3.0.3. Define the symbol class $\mathbb{S}_{S}^{1 / 2}$ by

$$
\begin{aligned}
\mathbb{S}_{S}^{1 / 2}:= & \left\{a \sim \sum_{j=0}^{+\infty} a_{1 / 2-j / 2} \in S_{S}^{1 / 2} \text { such that: } a_{1 / 2-j / 2}(x, \tau):=\right. \\
& \left.\frac{1}{\left(\lambda_{1 / 2}^{+}\right)^{j-1}} \sum_{\ell=0}^{L_{j}} \frac{\mathcal{F}_{\ell}^{V, 1 / 2-j / 2}}{\left(\lambda_{1 / 2}^{+}\right)^{\ell}}, \text { with } \mathcal{F}_{\ell}^{V, 1 / 2-j / 2} \in \mathcal{C}^{\infty}(\mathbb{R} ; \mathbb{R}), L_{j} \in \mathbb{N}\right\},
\end{aligned}
$$

and the associated class of pseudo-differential operators $\operatorname{OPS}_{S}^{1 / 2}$. In $(3.6), \mathcal{F}_{\ell}^{V, 1 / 2-j / 2}$ are smooth functions depending on $x$ and $V$. Then, $\Lambda^{ \pm}$are in $O P \mathbb{S}_{S}^{1 / 2}$ and, for each $j \in \mathbb{N}$, there exist some regular functions $\left\{\mathcal{F}_{\ell}^{V, 1 / 2-j / 2}\right\}_{\ell=0}^{L_{j}}$ such that

$$
\lambda_{1 / 2-j / 2}^{+}=-\lambda_{1 / 2-j / 2}^{-}=\frac{1}{\left(\lambda_{1 / 2}^{+}\right)^{j-1}} \sum_{\ell=0}^{L_{j}} \frac{\mathcal{F}_{\ell}^{V, 1 / 2-j / 2}}{\left(\lambda_{1 / 2}^{+}\right)^{\ell}} .
$$

Proof. For $j=0$, we trivially have

$$
\lambda_{1 / 2}^{+}=-\lambda_{1 / 2}^{-}=\frac{\mathcal{F}_{0}^{V, 1 / 2}}{\left(\lambda_{1 / 2}^{+}\right)^{-1}}
$$


with $\mathcal{F}_{0}^{V, 1 / 2}=1$ and $L_{0}=1$. Assume now that, for $0 \leq k \leq j$, with $k \in \mathbb{N}$, we have

$$
\lambda_{1 / 2-k / 2}^{ \pm}=\frac{1}{\left(\lambda_{1 / 2}^{+}\right)^{k-1}} \sum_{\ell=0}^{L_{k}} \frac{\mathcal{F}_{\ell}^{V, 1 / 2-k / 2}}{\left(\lambda_{1 / 2}^{+}\right)^{\ell}}
$$

We need to prove inductively that the expression (3.7) still holds for $j+1$. First, from (3.9), a direct calculation leads to

$$
\begin{aligned}
& \partial_{x} \lambda_{1-j / 2}^{+}=\partial_{x}\left(\sum_{\ell=0}^{L_{j}} \frac{\mathcal{F}_{\ell}^{V, 1 / 2-j / 2}}{\left(\lambda_{1 / 2}^{+}\right)^{j+\ell-1}}\right)= \\
& \sum_{\ell=0}^{L_{j}}\left(\frac{(1-j-\ell)}{2} \partial_{x} V \frac{\mathcal{F}_{\ell}^{V, 1 / 2-j / 2}}{\left(\lambda_{1 / 2}^{+}\right)^{j+\ell}}+\frac{\partial_{x} \mathcal{F}_{\ell}^{V, 1 / 2-j / 2}}{\left(\lambda_{1 / 2}^{+}\right)^{j+\ell-1}}\right)=\frac{1}{\left(\lambda_{1 / 2}^{+}\right)^{j-1}} \sum_{\ell=0}^{L_{j}} \frac{\mathcal{G}_{\ell}^{V, 1 / 2-j / 2}}{\left(\lambda_{1 / 2}^{+}\right)^{\ell}},
\end{aligned}
$$

for some given functions $\mathcal{G}_{\ell}^{V, 1 / 2-j / 2}$. Now, we also have

$$
\lambda_{-j / 2+k / 2}^{+}=\lambda_{1 / 2-(j-k+1) / 2}^{+}=\frac{1}{\left(\lambda_{1 / 2}^{+}\right)^{j-k}} \sum_{\ell=0}^{L_{j-k+1}} \frac{\mathcal{F}_{\ell}^{V,(k-j) / 2}}{\left(\lambda_{1 / 2}^{+}\right)^{\ell}}
$$

Consequently, we can deduce that

$$
\begin{aligned}
& \lambda_{1 / 2-k / 2}^{+} \lambda_{-j / 2+k / 2}^{+}=\left(\frac{1}{\left(\lambda_{1 / 2}^{+}\right)^{k-1}} \sum_{\ell=0}^{L_{k}} \frac{\mathcal{F}_{\ell}^{V,(1-k) / 2}}{\left(\lambda_{1 / 2}^{+}\right)^{\ell}}\right)\left(\frac{1}{\left(\lambda_{1 / 2}^{+}\right)^{j-k}} \sum_{\ell=0}^{L_{j-k+1}} \frac{\mathcal{F}_{\ell}^{V,(k-j) / 2}}{\left(\lambda_{1 / 2}^{+}\right)^{\ell}}\right) \\
& =\frac{1}{\left(\lambda_{1 / 2}^{+}\right)^{j-1}}\left(\sum_{\ell=0}^{L_{k}} \frac{\mathcal{F}_{\ell}^{V,(1-k) / 2}}{\left(\lambda_{1 / 2}^{+}\right)^{\ell}}\right)\left(\sum_{\ell=0}^{L_{j-k+1}} \frac{\mathcal{F}_{\ell}^{V,(k-j) / 2}}{\left(\lambda_{1 / 2}^{+}\right)^{\ell}}\right)=\frac{1}{\left(\lambda_{1 / 2}^{+}\right)^{j-1}} \sum_{\ell=0}^{L_{k}+L_{j-l+1}} \frac{\mathcal{H}_{\ell}^{V,(j, k)}}{\left(\lambda_{1 / 2}^{+}\right)^{\ell}}
\end{aligned}
$$

for some functions $\mathcal{H}_{\ell}^{V,(j, k)}$ which can be expressed thanks to $\mathcal{F}_{\ell}^{V,(1-k) / 2}$ and $\mathcal{F}_{\ell}^{V,(k-j) / 2}$. Now, looking at (3.5), we see that, by summing up the different expressions related to (3.10) and (3.12), we have

$$
\lambda_{-j / 2}^{+}=\frac{1}{\left(\lambda_{1 / 2}^{+}\right)^{j}} \sum_{\ell=0}^{L_{j+1}} \frac{\mathcal{F}_{\ell}^{V,-j / 2}}{\left(\lambda_{1 / 2}^{+}\right)^{\ell}}
$$

showing that the result holds for the index $j+1$. The functions $\mathcal{F}_{\ell}^{V,-j / 2}$ and the index $L_{j+1}$ 
can be deduced from the calculations if required. Proving that $\lambda_{1 / 2-j / 2}^{+}=-\lambda_{1 / 2-j / 2}^{-}$is a direct consequence of the results stated in [3] for Proposition (3.0.1).

\subsubsection{Asymptotic estimates of the contraction factor of the CSWR algorithm}

In this section the CSWR described in (2.4), is considered. The time-dependent functions $h_{\epsilon}^{ \pm}$are assumed to be given. In order to simplify the notation, we introduce $h_{\epsilon}^{ \pm}$the extension of $h_{\epsilon}^{ \pm}$to all $\mathbb{R}$ which vanishes on $\mathbb{R}_{-}$. As proposed in [8], we are required to determine the contraction factor $C_{P, \epsilon}^{C}$ of $\mathcal{G}_{P}^{C 2}$ (setting $\mathcal{G}_{P}^{C 2}:=\mathcal{G}_{P}^{C} \circ \mathcal{G}_{P}^{C}$ ), where the mapping $\mathcal{G}_{P}^{C}$ is defined by

$$
\mathcal{G}_{P}^{C}:\left\langle h_{\epsilon}^{+}, h_{\epsilon}^{-}\right\rangle \mapsto\left\langle e_{P}^{C,-}(\epsilon / 2, \cdot), e_{P}^{C,+}(-\epsilon / 2, \cdot)\right\rangle
$$

We solve (2.3) directly to prove that $\mathcal{G}_{P}^{C 2}$ is a contraction in the $(x, \tau)$-coordinates for $V=0$. For $V \neq 0$ (in fact, for a non-constant potential $V$ ), we estimate the rate of convergence through approximations. Let us start by assuming that $V$ is a constant (this includes $V=0$ ). According to [8], for a fixed time $T, \mathcal{G}_{P}^{C}$ is defined by $H_{0}^{3 / 4}(0, T)=\left\{\phi \in H^{3 / 4}(0, T): \phi(0)=\right.$

$0\}$. Let us characterize the part of the error $e_{P}^{C,+}$ (respectively $e_{P}^{C,-}$ ) which is a traveling wave in the overlapping region related to $\Omega_{\varepsilon}^{+}$(respectively $\Omega_{\varepsilon}^{-}$) and transmitted to the left (respectively right) domain $\mathbb{R} / \overline{\Omega_{\varepsilon}^{+}}$(respectively $\mathbb{R} / \overline{\Omega_{\varepsilon}^{-}}$). We next introduce the system of equations

$$
\left\{\begin{array}{l}
\left(\partial_{x}+i \Lambda^{\mp}\right) e_{\Lambda}^{C, \pm}=0, \text { in } \Omega_{\varepsilon}^{ \pm}, \\
e_{\Lambda}^{C, \pm}( \pm \epsilon / 2, t)=h_{\epsilon}^{ \pm}(t) \text { at }\{ \pm \epsilon / 2\} \times \mathbb{R} .
\end{array}\right.
$$

For $V=0$, the solution of the first equation of system (3.15) can be made explicitly and exactly through the Fourier transform $\mathcal{F}_{t}$ along the $t$-direction, that is, at the symbol level with respect to $\tau$. As seen below, if $V \neq 0$, this leads to an explicit but approximate solution. The exact solution to system (3.15) is given in the $(x, \tau)$-space by

$$
\hat{e}_{\Lambda}^{C, \pm}(x, \tau)=\hat{h}_{\epsilon}^{ \pm}(\tau) \exp \left(-i \int_{ \pm \epsilon / 2}^{x} \lambda^{\mp}(\tau) d y\right)
$$


We need to use the symbols for the real-time equation and we obtain these symbols for (2.1) from [3]. As a consequence, the application of Proposition (3.0.2) for $V$ being a constant leads to: $\lambda^{ \pm}(\tau)=\lambda_{1 / 2}^{ \pm}(\tau)=\mp \sqrt{-\tau+V}$. If we define

$$
\mathcal{G}_{\Lambda}^{C}:\left\langle h_{\epsilon}^{+}, h_{\epsilon}^{-}\right\rangle \mapsto\left\langle e_{\Lambda}^{C,-}(\epsilon / 2, \cdot), e_{\Lambda}^{C,+}(-\epsilon / 2, \cdot)\right\rangle
$$

we have

$$
\begin{aligned}
& \mathcal{F}_{t}\left(\mathcal{G}_{\Lambda}^{C 2}\left\langle h_{\epsilon}^{+}, h_{\epsilon}^{-}\right\rangle\right) \\
& =\left\langle\exp \left(i \int_{-\epsilon / 2}^{\epsilon / 2}\left(\lambda^{-}(\tau)-\lambda^{+}(\tau)\right) d y\right) \hat{h}_{\epsilon}^{+}, \exp \left(i \int_{-\epsilon / 2}^{\epsilon / 2}\left(\lambda^{-}(\tau)-\lambda^{+}(\tau)\right) d y\right) \hat{h}_{\epsilon}^{-}\right\rangle \\
& =\exp \left(-2 i \epsilon \lambda^{+}(\tau)\right)\left\langle\hat{h}_{\epsilon}^{+}, \hat{h}_{\epsilon}^{-}\right\rangle .
\end{aligned}
$$

Following [8], we deduce that the contraction factors $C_{\Lambda, \epsilon}^{C}$ of $\mathcal{G}_{\Lambda}^{C 2}$ and $C_{P, \epsilon}^{C}$ of $\mathcal{G}_{P}^{C 2}$ are the same and that

$$
C_{P, \epsilon}^{C}=C_{\Lambda, \epsilon}^{C}=\sup _{\tau \in \mathcal{H}_{\tau}} L_{\Lambda, \epsilon}^{C}(\tau)
$$

where

$$
L_{\Lambda, \epsilon}^{C}(\tau)=\left|\exp \left(-2 i \epsilon \lambda^{+}(\tau)\right)\right|=\exp (2 \epsilon i \sqrt{-\tau+V})
$$

In the above expression, $\mathcal{H}_{\tau}$ denoted the hyperbolic region $\{\tau \in \mathbb{R}, \tau<V\}$. For the elliptic region $\mathcal{E}_{\tau}=\{\tau \in \mathbb{R}: \tau>V\}$, one gets a similar expression. Taking the real part leads to an appropriate contraction factor. The contraction factors $C_{\Lambda, \epsilon}^{C}$ of $\mathcal{G}_{\Lambda}^{C 2}$ and $C_{P, \epsilon}^{C}$ of $\mathcal{G}_{P}^{C 2}$ are

$$
C_{P, \epsilon}^{C}=C_{\Lambda, \epsilon}^{C}=\sup _{\tau \in \mathcal{E}_{\tau}} L_{\Lambda, \epsilon}^{C}(\tau)
$$


where

$$
L_{\Lambda, \epsilon}^{C}(\tau)=\left|\exp \left(-2 i \epsilon \lambda^{+}(\tau)\right)\right|=\exp (2 i \epsilon \sqrt{-\tau+V})
$$

It is also noteworthy, the CSWR diverges without an overlapping region, that is, when $\epsilon=0$.

We will now consider the space-dependent potential $V(x)$. This means that the hyperbolic zone is now defined by $\{\tau \in \mathbb{R}: \tau<V(x)\}$. We need to characterize the error that travels from one domain to the other, as we performed above. However, the general potential $V(x)$ cannot be exact because of the scattering effects, due to $V(x)$, must be taken into account. Therefore, we still consider the system

$$
\left\{\begin{array}{l}
\left(\partial_{x}+i \Lambda^{\mp}\right) e_{\Lambda}^{C, \pm}=0, \text { in } \Omega_{\varepsilon}^{ \pm} \\
e_{\Lambda}^{C, \pm}( \pm \epsilon / 2, t)=h_{\epsilon}^{ \pm}(t) \text { at }\{ \pm \epsilon / 2\} \times \mathbb{R}
\end{array}\right.
$$

but $e_{\Lambda}^{C,+}$ (respectively $e_{\Lambda}^{C,-}$ ) must be understood as the part of $e_{P}^{C,+}$ (respectively $e_{P}^{C,-}$ ) which travels to the right (respectively left). As a consequence, the computation of $e_{\Lambda}^{C, \pm}$ provides an approximation of $e_{P}^{C, \pm}$ solution to $P e_{P}^{C, \pm}=0$. We can therefore obtain an approximation for $C_{P, \varepsilon}^{C}$ which is the contraction factor of $\mathcal{G}_{P}^{C 2}$ by $C_{\Lambda, \varepsilon}^{C}$ for $\mathcal{G}_{\Lambda}^{C 2}$

$$
C_{P, \varepsilon}^{C} \approx C_{\Lambda, \varepsilon}^{C}
$$

For solving (3.18), let us consider the equation at the symbol level, i.e.

$$
\left\{\begin{array}{l}
\left(\partial_{x}+i \lambda^{\mp}(x, \tau)\right) \hat{e}_{\Lambda}^{C, \pm}(x, \tau)=0, \text { in } \Omega_{\varepsilon}^{ \pm} \\
\hat{e}_{\Lambda}^{C, \pm}( \pm \epsilon / 2, \tau)=\hat{h}_{\epsilon}^{ \pm}(\tau) \text { at }\{ \pm \epsilon / 2\} \times \mathbb{R}
\end{array}\right.
$$

A direct computation gives

$$
\hat{e}_{\Lambda}^{C, \pm}(x, \tau)=\hat{h}_{\epsilon}^{ \pm}(\tau) \exp \left(-i \int_{ \pm \epsilon / 2}^{x} \lambda^{\mp}(y, \tau) d y\right)
$$


In order to be able to determine the contraction factor $C_{\Lambda, \epsilon}^{C}$ of the associated mapping $\mathcal{G}_{\Lambda}^{C 2}$ one requires the information of the total symbols $\lambda^{ \pm}$. However, for a general potential $V(x)$, this is generally impossible. We resolve this by asymptotic expansions $\left\{\lambda_{1 / 2-j / 2}^{ \pm}\right\}_{j=0}^{+\infty}$ of $\lambda^{ \pm}$. To get an estimate, we first expand $\lambda^{ \pm}$asymptotically as the sum of inhomogeneous symbols

$$
\lambda^{ \pm} \sim \sum_{j=0}^{ \pm \infty} \lambda_{1 / 2-j / 2}^{ \pm}
$$

and then we truncate up to the $(p+1)$ first terms

$$
\lambda^{ \pm} \sim \lambda^{ \pm, p}=\sum_{j=0}^{p} \lambda_{1 / 2-j / 2}^{ \pm}
$$

as proposed in [2]. This means the approximate convergence rates are

$$
C_{P, \epsilon}^{C} \approx C_{\Lambda, \epsilon}^{C} \approx C_{\epsilon}^{C, p}:=\sup _{\tau \in \mathcal{H}_{\tau}} L_{\epsilon}^{C, p}(\tau)
$$

with

$$
L_{\epsilon}^{C, p}(\tau)=\left|\exp \left(i \int_{-\epsilon / 2}^{\epsilon / 2}\left(\lambda^{-, p}(y, \tau)-\lambda^{+, p}(y, \tau)\right) d y\right)\right| .
$$

Then we chooses the principal symbol $\lambda_{1 / 2}^{ \pm}=\mp \sqrt{-\tau+V(x)}$, then ones gets

$$
\lambda^{-, p}=-\lambda^{+, p}
$$

implying that (3.22) becomes

$$
L_{\epsilon}^{C, p}(\tau)=\left|\exp \left(-2 i \int_{-\epsilon / 2}^{\epsilon / 2} \lambda^{+, p}(y, \tau) d y\right)\right|
$$

Let us remark that (3.23) does not hold for $\lambda_{1 / 2}^{ \pm}=\mp \sqrt{-\tau}$ if $V(x) \neq 0[3]$, since $\lambda_{1 / 2}^{=}-\sqrt{-\tau}$ would give some symbols which are approximations of $\lambda_{1 / 2}^{=}-\sqrt{-\tau+V(x)}$ by using a secondorder truncated Taylor expansion when $|\tau| \rightarrow+\infty$. A third approximation step required to 
be able to determine each symbol $\lambda_{1 / 2-j / 2}^{ \pm}, j=0, \ldots, p$, in association to the small parameter $1 /|\tau|$ (high-frequency regime). Specifically, for each symbol $\lambda_{1 / 2-j / 2}^{ \pm}$, we consider its Taylor's expansion $\left(\lambda_{1 / 2-j / 2}^{ \pm}\right)_{(1-p) / 2}$ up to the order $1 /|\tau|^{(p-1) / 2}$

$$
\lambda^{ \pm, p} \approx \widetilde{\lambda}^{ \pm, p}=\sum_{j=0}^{p}\left(\lambda_{1 / 2-j / 2}^{ \pm}\right)_{(1-p) / 2}
$$

We then define

$$
\widetilde{L}_{\epsilon}^{C, p}(\tau)=\left|\exp \left(-2 i \int_{-\epsilon / 2}^{\epsilon / 2} \tilde{\lambda}^{+, p}(y, \tau) d y\right)\right|,
$$

and the associated high-frequency asymptotic convergence rate $\widetilde{C}_{\epsilon}^{C, p}$ such that

$$
C_{P, \epsilon}^{C} \approx C_{\Lambda, \epsilon}^{C} \approx \widetilde{C}_{\epsilon}^{C, p}:=\sup _{\tau \in \mathcal{H}_{\tau}} \widetilde{L}_{\epsilon}^{C, p}(\tau)
$$

Let us set

$$
\begin{aligned}
& L_{\epsilon, 1 / 2-j / 2}(\tau)=\left|\exp \left(-2 i \int_{-\epsilon / 2}^{\epsilon / 2} \lambda_{1 / 2-j / 2}^{+}(y, \tau) d y\right)\right| \\
& \widetilde{L}_{\epsilon, 1 / 2-j / 2}^{p}(\tau)=\left|\exp \left(-2 i \int_{-\epsilon / 2}^{\epsilon / 2}\left(\lambda_{1 / 2-j / 2}^{+}\right)_{(1-p) / 2}(y, \tau) d y\right)\right| .
\end{aligned}
$$

Therefore, we have

$$
L_{\epsilon}^{C, p}=\prod_{j=0}^{p} L_{\epsilon, 1 / 2-j / 2} \text { and } \widetilde{L}_{\epsilon}^{C, p}=\prod_{j=0}^{p} \widetilde{L}_{\epsilon, 1 / 2-j / 2}^{p}
$$

We now present one of the main theorems, which gives the contraction fractor for the CSWR with respect to the linear Schrödinger equation.

Theorem 3.0.1. Let $V(x)$ be a smooth potential and let us assume that the symbols are defined as in Proposition 3.0.2. An asymptotic estimate of the contraction factor of the mapping $\mathcal{G}_{P}^{C 2}$ defined by (3.14), for the CSWR algorithm (2.4), is given by

$$
C_{P, \epsilon}^{C} \approx C_{\epsilon}^{C, 3}=\sup _{\tau \in \mathcal{H}_{\tau}} L_{\epsilon}^{C, 3}(\tau)
$$


for $|\tau| \gg 1$, where $\mathcal{H}_{\tau}$ is the hyperbolic zone and

$$
L_{\epsilon}^{C}(\tau) \approx L_{\epsilon}^{C, 3}(\tau):=\left|\left(\frac{-\tau+V(-\epsilon / 2)}{-\tau+V(+\epsilon / 2)}\right)^{1 / 2}\right|\left|\exp \left(-2 i \int_{-\epsilon / 2}^{\epsilon / 2} \sqrt{-\tau+V(y)} d y\right)\right|
$$

In addition, one also gets the following approximation when the symbols are Taylorized

$$
C_{P, \epsilon}^{C} \approx \widetilde{C}_{\epsilon}^{C, 3}=\sup _{\tau \in \mathcal{H}_{\tau}} \widetilde{L}_{\epsilon}^{C, 3}(\tau)
$$

with

$$
L_{\epsilon}^{C}(\tau) \approx \widetilde{L}_{\epsilon}^{C, 3}(\tau):=\exp \left(-2 \epsilon \sqrt{-\tau}+\frac{1}{2 \sqrt{-\tau}} \int_{-\epsilon / 2}^{\epsilon / 2} V(y) d y\right)
$$

Proof. From (3.20), we have

$$
\hat{e}_{\Lambda}^{C, \pm}(x, \tau)=\hat{h}_{\epsilon}^{ \pm}(\tau) \exp \left(-i \int_{ \pm \epsilon / 2}^{x} \lambda^{\mp}(y, \tau) d y\right)
$$

This implies that we have the approximation

$$
\mathcal{F}_{t}\left(\mathcal{G}_{P}^{2 C}\left\langle h_{\epsilon}^{+}, h_{\epsilon}^{-}\right\rangle\right) \approx \exp \left(i \int_{-\epsilon / 2}^{\epsilon / 2}\left(\lambda^{-}(y, \tau)-\lambda^{+}(y, \tau)\right) d y\right)\left\langle\hat{h}_{\epsilon}^{+}, \hat{h}_{\epsilon}^{-}\right\rangle
$$

By using Proposition 3.0.2 for the real-time equation, one gets

$$
\lambda_{1 / 2}^{ \pm}(x, \tau)=\mp \sqrt{-\tau+V(x)}, \quad \lambda_{-1}^{ \pm}(x, \tau)=\mp \frac{1}{4-\tau+V(x)},
$$

$\lambda_{0}^{ \pm}=0$ and $\lambda_{-1 / 2}^{ \pm}(x, \tau)=0$. A direct computation leads to

$$
\begin{aligned}
& L_{\epsilon, 1 / 2}(\tau)=\left|\exp \left(-2 i \int_{-\epsilon / 2}^{\epsilon / 2} \sqrt{-\tau+V(y)} d y\right)\right|, \\
& L_{\epsilon,-1}(\tau)=\left|\exp \left(\frac{i}{2} \int_{-\epsilon / 2}^{\epsilon / 2} \frac{V^{\prime}(y)}{-\tau+V(y)} d y\right)\right|,
\end{aligned}
$$


and $L_{\epsilon, 0}=L_{\epsilon,-1 / 2}=1$. Some calculations show that

$$
L_{\epsilon,-1}(\tau)=\left|\left(\frac{-\tau+V(-\epsilon / 2)}{-\tau+V(+\epsilon / 2)}\right)^{1 / 2}\right|
$$

As a consequence, one gets

$$
L_{\epsilon}^{C, p}(\tau)=\left|\left(\frac{-\tau+V(-\epsilon / 2)}{-\tau+V(+\epsilon / 2)}\right)^{1 / 2} \| \exp \left(-2 i \int_{-\epsilon / 2}^{\epsilon / 2} \sqrt{-\tau+V(y)} d y\right)\right|
$$

Now, by using a Taylor's expansion

$$
\left(\lambda_{1 / 2}^{+}\right)_{-1}=\sqrt{-\tau}\left(1-\frac{V(x)}{2 \tau}\right)
$$

we have the following estimates $\widetilde{L}_{\epsilon, 0}^{3}=\widetilde{L}_{\epsilon,-1 / 2}^{3}=\widetilde{L}_{\epsilon,-1}^{3}=1$ and

$$
\widetilde{L}_{\epsilon, 1 / 2}^{3}=\left|\exp \left(-2 \epsilon \sqrt{-\tau}-\frac{1}{2 \sqrt{-\tau}} \int_{-\epsilon / 2}^{\epsilon / 2} V(y) d y\right)\right|
$$

The proof follows from $\widetilde{L}_{\epsilon}^{C, 3}=\widetilde{L}_{\epsilon, 1 / 2}^{3}$.

All the above expressions are for the real-time hyperbolic region $\mathcal{H}_{\tau}=\{\tau \in \mathbb{R}: \tau<V\}$

\subsubsection{Asymptotic estimates of the contraction factor in the OSWR algorithm}

We now consider the Optimized Schwarz Waveform Relaxation (OSWR) method [9] and study its rates of convergence as a function of the order of the transmitting boundary conditions which are used. If we assume that $\phi^{ \pm,(0)}(\mp \epsilon / 2, \cdot)$ and $\phi_{0}^{ \pm}$are some given functions, 
the OSWR algorithm reads as follows for iterations: $k \geq 1$

$$
\left\{\begin{array}{l}
P \phi^{ \pm,(k)}=0, \text { in } \Omega_{\epsilon}^{ \pm} \times \mathbb{R}_{+}^{*}, \\
\phi^{ \pm,(k)}(\cdot, 0)=\phi_{0}^{ \pm}, \text {in } \Omega_{\epsilon}^{ \pm} \\
\left(\partial_{x}+i \Lambda^{ \pm, p}\right) \phi^{ \pm,(k)}( \pm \epsilon / 2, \cdot)=\left(\partial_{x}+i \Lambda^{ \pm, p}\right) \phi^{\mp,(k-1)}( \pm \epsilon / 2, \cdot) \text { in } \mathbb{R}_{+}^{*}
\end{array}\right.
$$

where, we set: $\Lambda^{ \pm, p}=\operatorname{Op}\left(\lambda^{ \pm, p}\right)$, for $p=1 / 2,0,-1 / 2 \ldots$ as we similarly did in (3.25) Following [8], we formally define in $\Omega_{\epsilon}^{ \pm}$

$$
P e_{P}^{O, \pm, p}=0 \text { on } \Omega_{\epsilon}^{ \pm, p} \times \mathbb{R}_{+}^{*}, \quad\left(\partial_{x}+i \Lambda^{ \pm, p}\right) e_{P}^{O, \pm, p}( \pm \epsilon / 2, t)=h_{\epsilon}^{ \pm}(t) \text { on }\{ \pm \epsilon / 2\} \times \mathbb{R}_{+}^{*},
$$

and we introduce the mapping

$$
\mathcal{G}_{P}^{O, p}:\left\langle h_{\epsilon}^{+}, h_{\epsilon}^{-}\right\rangle \mapsto\left\langle\left(\partial_{x}+i \Lambda^{+, p}\right) e_{P}^{O,-, p}(\epsilon / 2, \cdot),\left(\partial_{x}+i \Lambda^{-, p}\right) e_{P}^{O,+, p}(-\epsilon / 2, \cdot)\right\rangle .
$$

We again want to determine the asymptotic estimates of the convergence rate of the OSWR domain decomposition method when considering system (3.38) for a transmission condition $\lambda^{ \pm, p}$. The OSWR method has the following approximate boundary-value problem:

$$
\left\{\begin{array}{l}
\left(\partial_{x}+i \Lambda^{\mp}\right) e_{\Lambda}^{O, \pm, p}=0, \text { in } \Omega_{\varepsilon}^{ \pm} \times \mathbb{R}_{+}^{*} \\
\left(\partial_{x}+i \Lambda^{ \pm, p}\right) e_{\Lambda}^{O, \pm, p}( \pm \epsilon / 2, t)=h_{\epsilon}^{ \pm}(t), \text { on }\{ \pm \epsilon / 2\} \times \mathbb{R}_{+}^{*}
\end{array}\right.
$$

Then, the following remains valid as seen in [4], which leads to the next main theorem.

Theorem 3.0.2. Let us assume that $V$ is a smooth one-dimensional spatial-dependent potential and that $\lambda^{ \pm}$is approximated by

$$
\lambda^{ \pm} \approx \lambda^{ \pm, p}:=\sum_{j=0}^{p} \lambda_{1 / 2-j / 2}^{ \pm}
$$

with $|\tau| \gg 1$ and $p \in \mathbb{N}^{*}$. An asymptotic estimate of the contraction factor $C_{P, \epsilon}^{O}$ of the mapping $\mathcal{G}_{P}^{O, p 2}$ (with $\mathcal{G}_{P}^{O, p 2}=\mathcal{G}_{P}^{O, p} \circ \mathcal{G}_{P}^{O, p}$ ), defined by (3.40), for the fixed-point OSWR 
algorithm (3.38) is given by

$$
C_{P, \epsilon}^{O} \approx C_{\epsilon}^{O, p}=\sup _{\tau \in \mathcal{H}_{\tau}} L_{\epsilon}^{O, p}(\tau)
$$

where

$$
L_{\epsilon}^{O, p}(\tau) \approx c_{\epsilon}^{p} \frac{1}{\left|\lambda_{1 / 2}^{+}(\epsilon / 2, \tau) \lambda_{1 / 2}^{+}(-\epsilon / 2, \tau)\right|^{p+1}} L_{\epsilon}^{C, p}(\tau) .
$$

In the previous expression, $c_{\epsilon}^{p}$ is an $(\epsilon, p, V)$-dependent positive real-valued constant. The principal symbol is given by (3.3) and $L_{\epsilon}^{C, p}(\tau)$ designates the estimate of the convergence rate of the CSWR method given by equation (3.24). For a constant potential $V$, the fixed point OSWR algorithm (3.38) converges in two iterations for the one-dimensional potentialfree case, even without overlap $(\epsilon=0)$.

Proof. We introduce the approximate problem (3.41) which gives us the approximate representation $\hat{e}_{\Lambda}^{O, \pm, p}$ for the error $\hat{e}_{P}^{O, \pm, p}$. Similar to the CSWR method, the aim is to derive the convergence rate of the operator (later denoted $\mathcal{G}_{\Lambda}^{O, p}$, see (3.45)) analogous to problem (3.41) to approximate the exact rate of convergence of the application $\mathcal{G}_{P}^{O, p}$ given by (3.40) for the initial problem (3.39) for the OSWR approach. This can be expressed at the symbol level and we know from (3.41)

$$
\left\{\begin{array}{l}
\left(\partial_{x}+i \lambda^{\mp}\right) \hat{e}_{\Lambda}^{O, \pm, p}=0, \text { in } \Omega_{\varepsilon}^{ \pm} \times \mathbb{R}_{+}^{*}, \\
\left(\partial_{x}+i \lambda^{ \pm, p}\right) \hat{e}_{\Lambda}^{O, \pm, p}( \pm \epsilon / 2, t)=\hat{h}_{\epsilon}^{ \pm}(t), \text { on }\{ \pm \epsilon / 2\} \times \mathbb{R}_{+}^{*},
\end{array}\right.
$$

which leading to the following expression

$$
\hat{e}_{\Lambda}^{O, \pm, p}(x, \tau)=\alpha_{\epsilon}^{ \pm, p}(\tau) \exp \left(-i \int_{ \pm \epsilon / 2}^{x} \lambda^{\mp}(y, \tau) d y\right)
$$

for some functions $\alpha_{\epsilon}^{ \pm, p}$. By implementing the transmitting boundary conditions (second 
equation of system (3.43)), we get the following

$$
\hat{e}_{\Lambda}^{O, \pm, p}(x, \tau)=\frac{\hat{h}_{\epsilon}^{ \pm}(\tau)}{i\left(\lambda^{ \pm, p}( \pm \epsilon / 2, \tau)-\lambda^{\mp}( \pm \epsilon / 2, \tau)\right)} \exp \left(-i \int_{ \pm \epsilon / 2}^{x} \lambda^{\mp}(y, \tau) d y\right) .
$$

Now we consider the mapping

$$
\mathcal{G}_{\Lambda}^{O, p}:\left\langle h_{\epsilon}^{+}, h_{\epsilon}^{-}\right\rangle \mapsto\left\langle\left(\partial_{x}+i \Lambda^{+, p}\right) \cdot e_{\Lambda}^{O,-, p}(\epsilon / 2, \cdot),\left(\partial_{x}+i \Lambda^{-, p}\right) \cdot e_{\Lambda}^{O,+, p}(-\epsilon / 2, \cdot)\right\rangle .
$$

We can therefore deduce that

$$
\begin{aligned}
& \mathcal{F}_{t}\left\langle\mathcal{G}_{\Lambda}^{O, p}\left(h_{\epsilon}^{+}, h_{\epsilon}^{-}\right)\right\rangle=\left\langle\left(\partial_{x}+i \lambda^{+, p}\right) \hat{e}_{\Lambda}^{O,-, p}(\epsilon / 2, \cdot),\left(\left(\partial_{x}+i \lambda^{-, p}\right) \hat{e}_{\Lambda}^{O,+, p}(-\epsilon / 2, \cdot)\right\rangle\right. \\
& =i\left\langle\left(\lambda^{+, p}(\epsilon / 2, \tau)-\lambda^{+}(\epsilon / 2, \tau)\right) \hat{e}_{\Lambda}^{O,-, p}(\epsilon / 2, \cdot),\left(\lambda^{-, p}(-\epsilon / 2, \tau)-\lambda^{-}(-\epsilon / 2, \tau)\right) \hat{e}_{\Lambda}^{O,+, p}(-\epsilon / 2, \cdot)\right\rangle \\
& =i\left\langle\sum_{j=p+1}^{+\infty} \lambda_{1 / 2-j / 2}^{-}(\epsilon / 2, \tau) \hat{e}_{\Lambda}^{O,-, p}(\epsilon / 2, \cdot), \sum_{j=p+1}^{+\infty} \lambda_{1 / 2-j / 2}^{+}(-\epsilon / 2, \tau) \hat{e}_{\Lambda}^{O,+, p}(-\epsilon / 2, \cdot)\right\rangle .
\end{aligned}
$$

To be able to determine an explicit expression of $\mathcal{F}_{t}\left(\mathcal{G}_{\Lambda}^{O, p 2}\left\langle h_{\epsilon}^{+}, h_{\epsilon}^{-}\right\rangle\right)$, another iterations required by fixing the boundary data $h_{\epsilon}^{ \pm,(2)}$ to

$$
\hat{h}_{\epsilon}^{ \pm, p,(2)}(\tau)=i\left(\lambda^{\mp}( \pm \epsilon / 2, \tau)+\lambda^{ \pm, p}( \pm \epsilon / 2, \tau)\right) \hat{e}_{\Lambda}^{O, \mp, p}( \pm \epsilon / 2, \tau)
$$

and then

$\hat{e}_{\Lambda}^{O, \pm, p,(2)}(x, \tau)=\frac{\lambda^{\mp}( \pm \epsilon / 2, \tau)+\lambda^{ \pm, p}( \pm \epsilon / 2, \tau)}{\lambda^{ \pm, p}( \pm \epsilon / 2, \tau)-\lambda^{\mp}( \pm \epsilon / 2, \tau)} \hat{e}_{\Lambda}^{O, \mp, p}( \pm \epsilon / 2, \tau) \exp \left(-i \int_{ \pm \epsilon / 2}^{x} \lambda^{\mp}(y, \tau) d y\right)$. 
By using (3.44), we get

$$
\begin{aligned}
\hat{e}_{\Lambda}^{O, \pm, p,(2)}(x, \tau)= & \frac{\lambda^{\mp}( \pm \epsilon / 2, \tau)+\lambda^{ \pm, p}( \pm \epsilon / 2, \tau)}{\lambda^{ \pm, p}( \pm \epsilon / 2, \tau)-\lambda^{\mp}( \pm \epsilon / 2, \tau)} \times \frac{\hat{h}_{\epsilon}^{\mp}(\tau)}{i\left(\lambda^{\mp, p}(\mp \epsilon / 2, \tau)-\lambda^{ \pm}(\mp \epsilon / 2, \tau)\right)} \\
& \times \exp \left(-i \int_{\mp \epsilon / 2}^{ \pm \epsilon / 2} \lambda^{ \pm}(y, \tau) d y\right) \exp \left(-i \int_{ \pm \epsilon / 2}^{x} \lambda^{\mp}(y, \tau) d y\right) .
\end{aligned}
$$

Now, we have

$$
\begin{aligned}
\mathcal{F}_{t}\left(\mathcal{G}_{\Lambda}^{O, p} \circ \mathcal{G}_{\Lambda}^{O, p}\left\langle h_{\epsilon}^{+}, h_{\epsilon}^{-}\right\rangle\right)(\tau) & \\
& =\left\langle\left(\partial_{x}+i \lambda^{+, p}\right) \hat{e}_{\Lambda}^{O,-, p,(2)}(\epsilon / 2, \cdot),\left(\partial_{x}+i \lambda^{-, p}\right) \hat{e}_{\Lambda}^{O,+, p,(2)}(-\epsilon / 2, \cdot)\right\rangle .
\end{aligned}
$$

It is then necessary to evaluate $\left(\partial_{x}+i \lambda^{ \pm, p}\right) \hat{e}_{\Lambda}^{O, \mp, p,(2)}( \pm \epsilon / 2, \cdot)$ through

$$
\begin{aligned}
\left(\partial_{x}+\right. & \left.i \lambda^{ \pm, p}\right) \cdot \hat{e}_{\Lambda}^{O, \mp, p,(2)}( \pm \epsilon / 2, \cdot) \\
& =\frac{\lambda^{\mp}( \pm \epsilon / 2, \tau)+\lambda^{ \pm, p}( \pm \epsilon / 2, \tau)}{\lambda^{ \pm, p}( \pm \epsilon / 2, \tau)-\lambda^{\mp}( \pm \epsilon / 2, \tau)} \times \frac{\lambda^{\mp, p}(\mp \epsilon / 2, \tau)-\lambda^{\mp}(\mp \epsilon / 2, \tau)}{\lambda^{\mp, p}(\mp \epsilon / 2, \tau)-\lambda^{ \pm}(\mp \epsilon / 2, \tau)} \\
& \times \exp \left(-2 i \int_{\mp \epsilon / 2}^{ \pm \epsilon / 2} \lambda^{ \pm}(y, \tau) d y\right) \hat{h}_{\epsilon}^{ \pm}(\tau) .
\end{aligned}
$$

Recall that $\lambda^{-}=-\lambda^{+}$and $\lambda^{-, p}=-\lambda^{+, p}$, therefore, we can simplify the above expression as

$$
\begin{aligned}
\left(\partial_{x}+\right. & \left.i \lambda^{ \pm, p}\right) \cdot \hat{e}_{\Lambda}^{O, \mp, p,(2)}( \pm \epsilon / 2, \cdot) \\
& =\frac{\lambda^{+, p}( \pm \epsilon / 2, \tau)-\lambda^{+}( \pm \epsilon / 2, \tau)}{\lambda^{+, p}( \pm \epsilon / 2, \tau)+\lambda^{+}( \pm \epsilon / 2, \tau)} \times \frac{\lambda^{+}(\mp \epsilon / 2, \tau)-\lambda^{+, p}(\mp \epsilon / 2, \tau)}{\lambda^{+, p}(\mp \epsilon / 2, \tau)+\lambda^{+}(\mp \epsilon / 2, \tau)} \\
& \times \exp \left(-2 i \int_{-\epsilon / 2}^{\epsilon / 2} \lambda^{+}(y, \tau) d y\right) \hat{h}_{\epsilon}^{\mp}(\tau) .
\end{aligned}
$$


Based on (3.47) and by using (3.49), one deduces that

$$
\begin{aligned}
& \mathcal{F}_{t}\left(\mathcal{G}_{\Lambda}^{O, p} \circ \mathcal{G}_{\Lambda}^{O, p}\left\langle h_{\epsilon}^{+}, h_{\epsilon}^{-}\right\rangle\right)(\tau) \\
& =\frac{\left[\lambda^{+, p}(\epsilon / 2, \tau)-\lambda^{+}(\epsilon / 2, \tau)\right]\left[\lambda^{+}(-\epsilon / 2, \tau)-\lambda^{+, p}(-\epsilon / 2, \tau)\right]}{\left[\lambda^{+, p}(\epsilon / 2, \tau)+\lambda^{+}(\epsilon / 2, \tau)\right]\left[\lambda^{+}(-\epsilon / 2, \tau)+\lambda^{+, p}(-\epsilon / 2, \tau)\right]} \\
& \times \exp \left(-2 i \int_{-\epsilon / 2}^{\epsilon / 2} \lambda^{+}(y, \tau) d y\right)\left\langle\hat{h}_{\epsilon}^{+}, \hat{h}_{\epsilon}^{-}\right\rangle(\tau) .
\end{aligned}
$$

Notice that $\lambda^{+, p}$ coincides with $\lambda^{+}$when " $p=\infty$ ", which formally induced that the exact convergence will result in two iterations since the numerator in (3.50) becomes null

$$
\mathcal{F}_{t}\left(\mathcal{G}_{\Lambda}^{O, p} \circ \mathcal{G}_{\Lambda}^{O, p}\left\langle h_{\epsilon}^{+}, h_{\epsilon}^{-}\right\rangle\right)(\tau)=0 \times\left\langle\hat{h}_{\epsilon}^{+}, \hat{h}_{\epsilon}^{+}\right\rangle(\tau)
$$

In the special case where $V$ is constant and consequently $e_{P}^{O, \pm}=e_{\Lambda}^{O, \pm}$, we also recover that the OSWR algorithm converges in two iterations [8]. Although, $p$ is a finite number in practice. We can derive the approximate rate of convergence $C_{\epsilon}^{O, p}$ of the OSWR algorithm from the exact convergence rate $C_{P, \epsilon}^{O}$ from (3.50). From Proposition 3.0.3, we know that we have the following asymptotic control (for large $|-\tau+V| \gg 1$ ) of the remaining term

$$
\begin{aligned}
& \lambda^{+}( \pm \epsilon / 2, \tau)-\lambda^{+, p}( \pm \epsilon / 2, \tau)=\sum_{j=p+1}^{+\infty} \lambda_{1 / 2-j / 2}^{+}( \pm \epsilon / 2, \tau) \\
& =\frac{1}{\left(\lambda_{1 / 2}^{+}( \pm \epsilon / 2, \tau)\right)^{p}} \sum_{j=p+1}^{+\infty} \sum_{\ell=0}^{L_{j+1}} \frac{\mathcal{F}_{\ell}^{V,-j / 2}}{\left(\lambda_{1 / 2}^{+}\right)^{\ell}}( \pm \epsilon / 2, \tau)=\mathcal{O}\left(\frac{1}{\left(\lambda_{1 / 2}^{+}( \pm \epsilon / 2, \tau)\right)^{p}}\right) .
\end{aligned}
$$

Furthermore, we have

$$
\lambda^{+}( \pm \epsilon / 2, \tau)+\lambda^{+, p}( \pm \epsilon / 2, \tau)=2 \lambda_{1 / 2}^{+}( \pm \epsilon / 2, \tau)+\mathcal{O}(1)
$$


Collecting the various estimates, we conclude that

$$
\begin{aligned}
& \mathcal{F}_{t}\left(\mathcal{G}_{\Lambda}^{O, p 2}\left\langle h_{\epsilon}^{+}, h_{\epsilon}^{-}\right\rangle\right)(\tau)= \\
& \mathcal{O}\left(\frac{1}{\left(\lambda_{1 / 2}^{+}(\epsilon / 2, \tau) \lambda_{1 / 2}^{+}(-\epsilon / 2, \tau)\right)^{p+1}}\right) \times \exp \left(-2 i \int_{-\epsilon / 2}^{\epsilon / 2} \lambda^{+}(y, \tau) d y\right)\left\langle h_{\epsilon}^{+}, h_{\epsilon}^{-}\right\rangle(\tau) .
\end{aligned}
$$

After truncating the symbolical expansion within the exponential term, an approximation rate of convergence for the OSWR DDM is given by

$$
C_{\epsilon}^{O, p}=\sup _{\tau \in \mathcal{H}_{\tau}} L_{\epsilon}^{O, p}(\tau)
$$

where

$$
L_{\epsilon}^{O, p}(\tau) \approx c_{\epsilon}^{p} \frac{1}{\left|\lambda_{1 / 2}^{+}(\epsilon / 2, \tau) \lambda_{1 / 2}^{+}(-\epsilon / 2, \tau)\right|^{p+1}} L_{\epsilon}^{C, p}(\tau)
$$

and $L_{\epsilon}^{C, p}(\tau)$ is given by (3.24), $p \in \mathbb{N}^{*}$, and $c_{\epsilon}^{p}$ is an $\epsilon$-, $p$ - and $V$-dependent positive real-valued constant.

\subsubsection{Convergence of the CSWR and OSWR algorithm}

In this section, we provide the convergence theorems of the CSWR (2.4) and OSWR (3.38) algorithms with $V$ constant, which were proved in [9] and rely on the calculation of a contraction factor. The following theorem provides the convergence for the CSWR algorithm.

Theorem 3.0.3. Let an initial guess $\left(h^{+}, h^{-}\right)$in $\left(H^{3 / 4}(0, T)\right)^{2}$ such that $h^{+}(0)=\phi_{0}(-\epsilon / 2)$ and $h^{-}(0)=\phi_{0}(\epsilon / 2)$. Let $V$ be a real constant and suppose $\tau_{\text {max }}>0$ such that $\mathcal{F}\left(e^{-t} h^{+}\right)$ and $\mathcal{F}\left(e^{-t} h^{-}\right)$vanish outside of $\left[-\tau_{\max },+\infty\right)$ with $g^{+}=h^{+}-\phi^{+}(-\epsilon / 2, \cdot)$ and $g^{-}=h^{-}-$ $\phi^{-}(\epsilon / 2, \cdot)$. Then the iterates $\left(\phi^{+,(k)}, \phi^{-,(k)}\right)$ of the algorithm $(2.4)$ converge in $L^{2}\left(\Omega_{\epsilon}^{+} \times\right.$ $(0, T)) \times L^{2}\left(\Omega_{\epsilon}^{-} \times(0, T)\right)$ to the solution of $(1.1)$.

The above theorem describes the convergence of the overlapping Schwarz waveform relaxation algorithm. Moreover, it demonstrates the convergence is at least linear and that 
it depends on the overlap $\Gamma_{\epsilon}$. For the CSWR, if the potential $V$ is constant, we have the approximate and the exact contraction factors coincide. That is,

$$
C_{P, \epsilon}^{C}=C_{\Lambda, \epsilon}^{C}
$$

The OSWR convergence theorem reads as follows:

Theorem 3.0.4. Let $V$ be a real constant. Algorithm (3.38) converges to the solution $\phi$ of (1.1) in two iterations for all initial guesses $\phi^{+,(0)}$ and $\phi^{-,(0)}$, independently of the size of the overlap $(-\epsilon / 2, \epsilon / 2)$ for $\epsilon>0$ if and only if the operator $P$ have the corresponding symbols

$$
\lambda^{+}=(-\tau-V)^{1 / 2}, \lambda^{-}=-(-\tau+V)^{1 / 2}
$$

When $V$ is constant, the OSWR algorithm then convergences in two iterations, which corresponds to a contraction fractor equal to 0 . When $V$ is non-constant, we expect a similar result, and thanks to our above analysis, at least at high frequencies, the contraction factor can be shown to be arbritrarily small. Note that, however, a rigurous proof of the convergence of the OSWR algorithm for $V$ non- constant has yet to be established (work in progress). We refer to [5] for numerical experiments on OSWR. 


\section{Chapter 4}

\section{Numerical Validation}

\subsubsection{Numerical approximation}

In the one-dimensional case, we consider 2 subdomains $\Omega_{a, \epsilon}^{+}=(-a, \epsilon / 2), \Omega_{a, \epsilon}^{-}=(-\epsilon / 2, a)$, $a \in \mathbb{R}_{+}^{*}$ with a (small) parameter $\epsilon>0$, characterizing the overlap region $\Gamma_{a, \epsilon}=\Omega_{a, \epsilon}^{+} \cap \Omega_{a, \epsilon}^{-}=$ $(-\epsilon / 2, \epsilon / 2)$, and $\Omega_{a}=\Omega_{\epsilon}^{+} \cup \Omega_{\epsilon}^{-}=(-a, a)$. In order to simplify the notation, we denote the solution $\phi^{+}$(respectively $\left.\phi^{-}\right)$in $\Omega_{a, \epsilon}^{+}$(respectively $\Omega_{a, \epsilon}^{+}$) by $u$ (respectively $v$ ). The numerical scheme which is used, is an unconditionally $\ell^{2}$-stable and order 2, Crank-Nicolson scheme $(\mathrm{CN})$. We denote $u_{j}^{n}$ and $v_{j}^{n}$ to be the solution of the scheme at iteration $n$ at $x_{j}$, where $x_{j}=a+j \Delta x$ We also denote by $\Delta x$ the space step and $\Delta t_{n}$ the time-step at iteration $n$. CN is defined as follows: for interior points in $(a, b)$ and for $1<j<N_{1}$ and Schwartz iteration $k \geq 1$

$$
\begin{aligned}
\frac{u_{j}^{n+1,(k)}-u_{j}^{n,(k)}}{\Delta t_{n}} & =\frac{i}{2 \Delta x^{2}}\left(u_{j+1}^{n+1,(k)}-2 u_{j}^{n+1,(k)}+u_{j-1}^{n+1,(k)}\right) \\
& +\frac{i}{2 \Delta x^{2}}\left(u_{j}^{n+1,(k)}-2 u_{j}^{n+1,(k)}+u_{j-1}^{n+1,(k)}\right)-\frac{i}{2} V_{j}\left(u_{j}^{n+1,(k)}+u_{j}^{n,(k)}\right),
\end{aligned}
$$


with Dirichlet boundary conditions $u_{N_{1}}^{n+1,(k)}=v_{j_{0}}^{n+1,(k-1)}$, where $V_{j}=V\left(x_{j}\right)$ and $j_{0}$ denotes the number of overlapping nodes. In $\Omega_{a, \epsilon}^{-}$, for $1<j<N_{2}$, we have

$$
\begin{aligned}
\frac{v_{j}^{n+1,(k)}-v_{j}^{n,(k)}}{\Delta t_{n}} & =\frac{i}{2 \Delta x^{2}}\left(v_{j+1}^{n+1,(k)}-2 v_{j}^{n+1,(k)}+v_{j-1}^{n+1,(k)}\right) \\
& +\frac{i}{2 \Delta x^{2}}\left(v_{j}^{n+1,(k)}-2 v_{j}^{n+1,(k)}+v_{j-1}^{n+1,(k)}\right),
\end{aligned}
$$

with Dirichlet boundary conditions $v_{1}^{n+1,(k)}=u_{N_{1}-j_{0}}^{n+1,(k-1)}$.

\subsubsection{Test 1: potential $V=0$}

The first test is devoted to 2 waves propagating in opposite direction from time 0 to final time $T=15$. We follow the experiment given in [5]. The parameters for the first computation

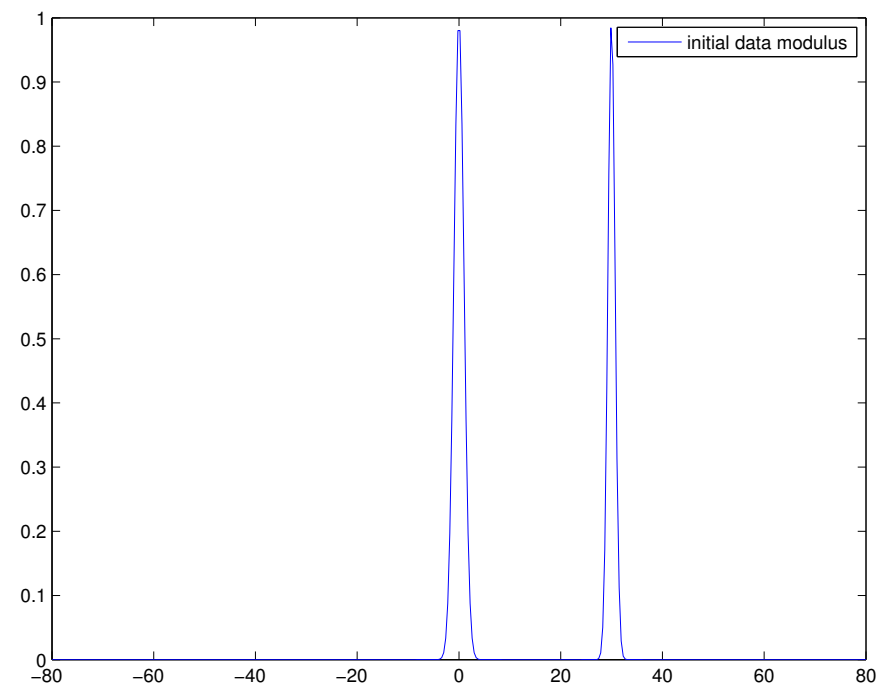

Figure 4-1: Initial data modulus.

are the following:

- $a=80, b=20$, with $N=800$. 
- Time step $\Delta t=0.2$.

- The overlapping region is reduced to two nodes, $j_{0}=2$, that is $\epsilon=\Delta x$.

- The initial data is given by

$$
u_{0}(x)=\exp \left(-\frac{1}{2}\left(\frac{b-a}{2}-x\right)^{2}\right) \exp \left(i k_{0} x\right)+\exp \left(-\left(\frac{3 a}{8}+x\right)^{2}\right) \exp \left(-i k_{0} x\right),
$$

with $k_{0}=4$. (see Figure (4-1)).

In Figure (4-2), we see an agreement the reconstructed solution using the CSWR with the $\mathrm{CN}$ scheme compared to the reference solution. We have that $\epsilon=\Delta x$ and time frequency $\left|\tau_{\text {num }}\right| \in[1 / T, 1 \Delta t]$, from the above analysis (3.18), we get in the elliptic region

$$
C_{P, \epsilon}^{C}=C_{\Lambda, \epsilon}^{C}=\sup _{\left|\tau_{\text {num }}\right| \in[1 / T, 1 \Delta t]} L_{\Lambda, \epsilon}^{C}\left(\tau_{\text {num }}\right)
$$

where

$$
L_{\Lambda, \epsilon}^{C}\left(\tau_{\text {num }}\right)=\left|\exp \left(-2 i \epsilon \lambda^{+}\left(\tau_{\text {num }}\right)\right)\right|=|\exp (2 i \Delta x \sqrt{-1 / T})|
$$

where the supremum is actually reached at final time T. Figure (4-2) also compares the
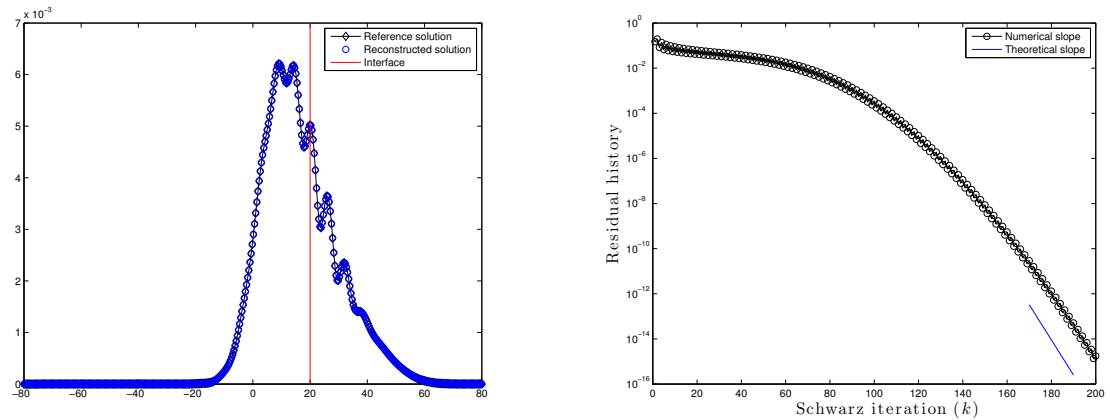

Figure 4-2: (Left) CSWR: reconstructed converged solution after iteration $k$ until convergence at final time $T$. (Right) Theoretical slope and numerical convergence rates comparison.

numerical convergence rate obtained by the $\mathrm{CN}$ scheme and the theoretical convergence rates 
(3.32). We observe a good agreement between the theoretical and the computed convergence rates. The comparison of varying time-steps $\Delta t$ is also provided in Figure (4-3). We observe
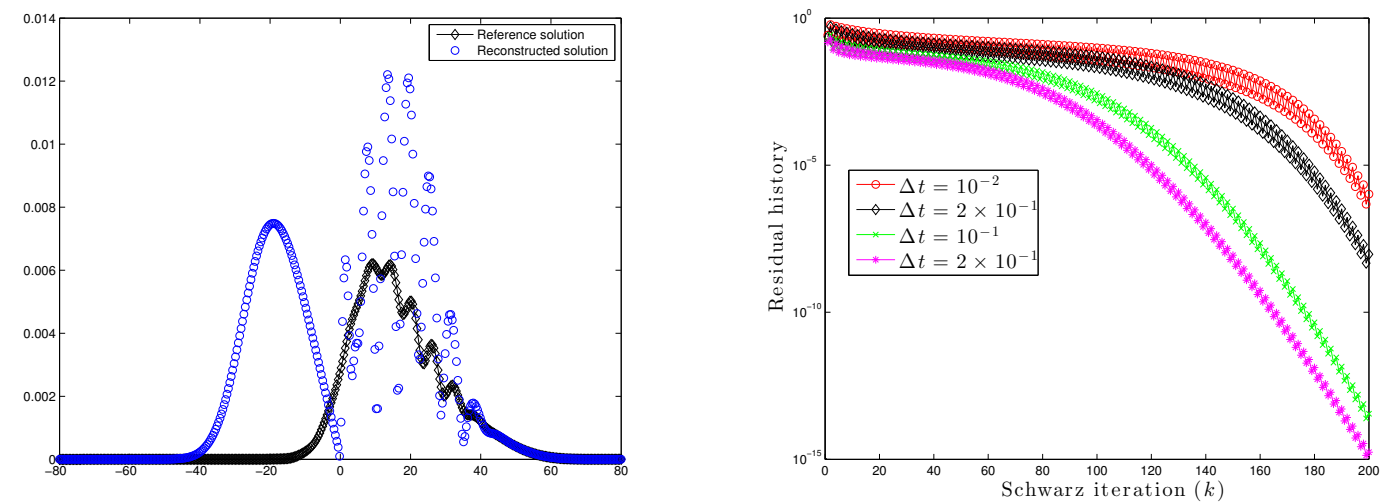

Figure 4-3: (Left) CSWR after 1 Schwarz iteration at final time T. (Right) Comparison between convergence rates with different time-steps $\Delta t$.

the convergence requires fewer iterations, when the time-steps $\Delta t$ are larger; however, notice that the convergence slope is independent of the time-step $\Delta t$, but dependent on final time $T$ (see Figure (4-4)). We also observe that the convergence is faster for smaller final time $T$ in Figure (4-4).

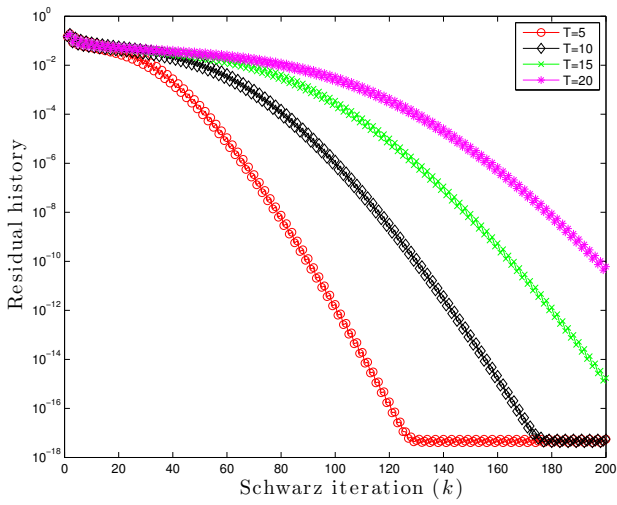

Figure 4-4: Comparison between convergence rates with different final times $T$. 


\subsubsection{Test 2: potential $V$}

The next test is also devoted to 2 waves propagating in opposite directions from time 0 to $T=15$ with a positive space-dependent potential $V$. We recall that this time,

$$
\begin{aligned}
& \sup _{\tau_{\text {num }} \in[1 / T, 1 / \Delta t]} L_{\Delta x}^{C}\left(\tau_{\text {num }}\right) \approx \\
& \left(\left|\left(\frac{\tau_{\text {num }}-V(-\Delta x / 2)}{\tau_{\text {num }}-V(+\Delta x / 2)}\right)^{1 / 2} \| \exp \left(-2 i \Delta x \int_{-\Delta x / 2}^{\Delta x / 2} \sqrt{-\tau_{\text {num }}+V(y)} d y\right)\right|\right),
\end{aligned}
$$

which can be further approximated by a Taylor's expansion following (3.32) as

$$
\sup _{\tau_{\text {num }} \in[1 / T, 1 / \Delta t]} L_{\Delta x}^{C}\left(\tau_{\text {num }}\right) \approx \exp \left(-\Delta x \sqrt{\tau_{\text {num }}}-V(b) \Delta x \sqrt{\frac{\tau_{\text {num }}}{2}}\right) .
$$

where the interface is located at $x=b$.

The parameters for the second experiment are as follows:

- $a=80, b=20$, with $N=800$.

- The time step $\Delta t=0.2$.

- The overlapping region is reduced to two nodes, $j_{0}=2$ such that $\epsilon=\Delta x$

- The initial data is given by

$$
u_{0}(x)=\exp \left(-\frac{1}{2}\left(\frac{b-a}{2}-x\right)^{2}\right) \exp \left(i k_{0} x\right)+\exp \left(-\left(\frac{-3 a}{8}-x\right)^{2}\right) \exp \left(-i k_{0} x\right)
$$

with $k_{0}=4$.

- The space dependent potential given by

$$
V(x)=5 \exp \left(-2 x^{2}\right)
$$

In Figure (4-5), we again observe a relatively good agreement between the numerical and 

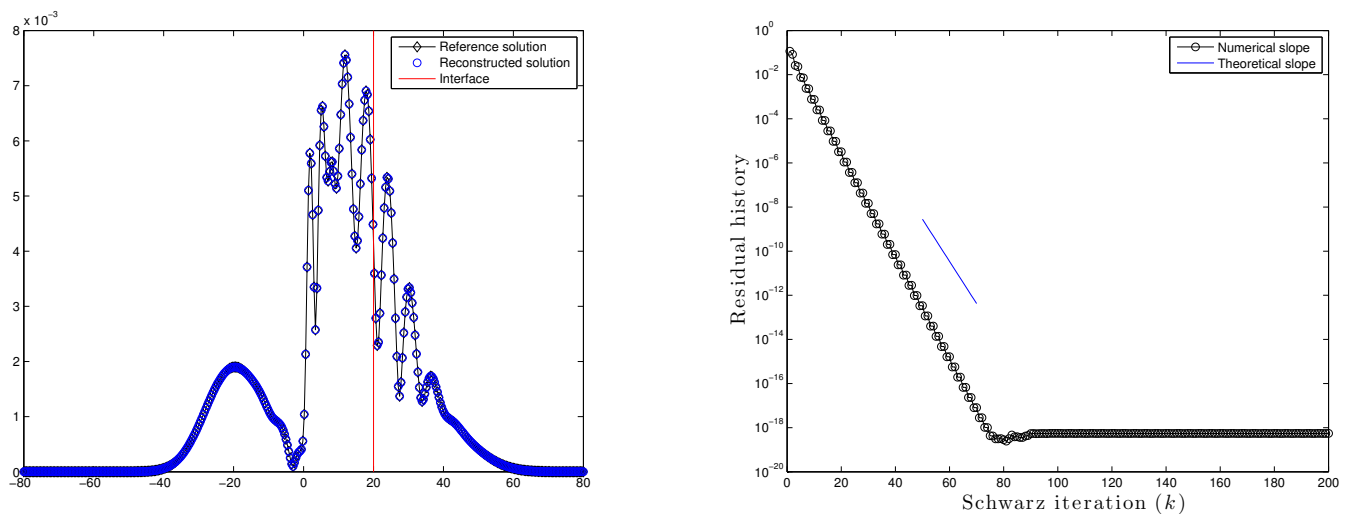

Figure 4-5: (Left): Comparison between the reconstructed solution and the reference solution with space dependent potential $V$. (Right) Theoretical slope and numerical convergence rates compared.

theoretical convergence rates. In Figure (4-6) comparisons are made between various timesteps $\Delta t$ and their associated convergence rates. We observe that, the larger the time-step, the faster the convergence. In Figure (4-6), one observes that after 1 Schwarz iteration with
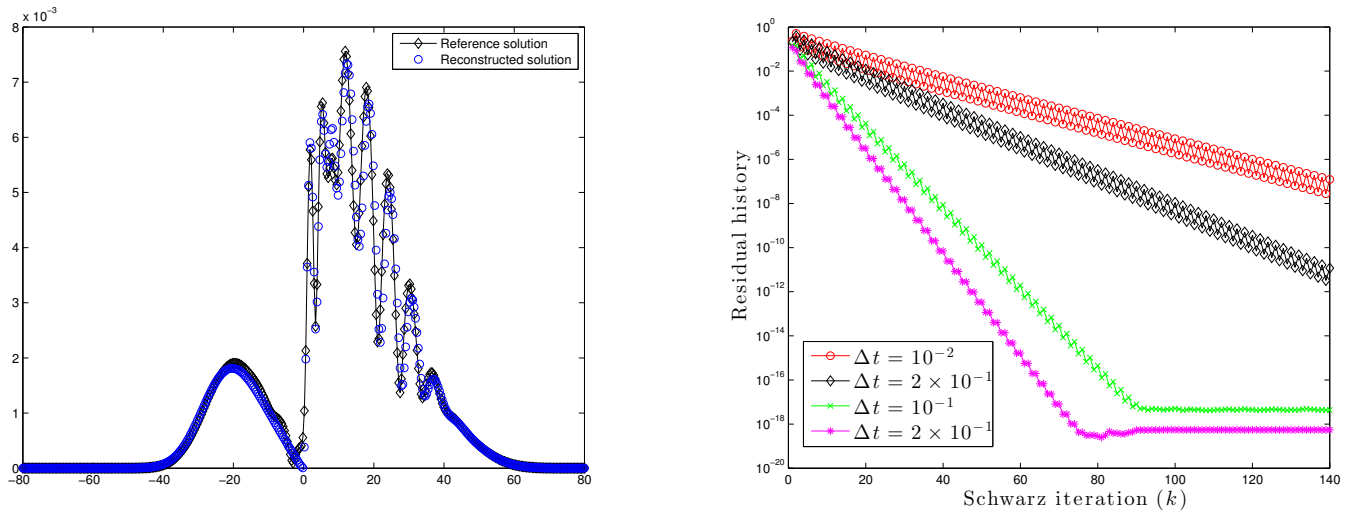

Figure 4-6: (Left) Comparison between reconstructed solution after 1 Schwarz iteration and the reference solution at final time $T$. (Right) Comparison between convergence rates with different time-steps $\Delta t$ and space dependent potential $V$ for the CSWR

space dependent potential $V$, we are close to convergence unlike the case $V=0$. This is because the convergence rate is improved by positive potentials. One can also notice the convergence rate depends on where the supremum of $\left|\tau_{\text {num }}\right| \in[1 / T, 1 / \Delta t]$ occurs, where in 
the null potential case, the supremum always occurs at final time $T$. 


\section{Chapter 5}

\section{Conclusion}

The thesis looked at the analysis of the classical (CSWR) and the optimized (OSWR) Schwarz Waveform Relaxation domain decomposition methods for parallel computation applied to the linear Schrödinger equation. In the analysis, we established some main theorems about the theoretical convergence rates. These theoretical convergence rates were computed for both the CSWR and the OSWR algorithms using pseudo-differential calculus and the associated asymptotic symbol expansions. The theoretical convergence rates for the CSWR algorithm were tested against the numerical convergence rates, and it was observed that they had good agreement. The proof of the well-posedness for the CSWR was also provided. 


\section{Bibliography}

[1] S. Alinhac and P. Gérard. Pseudo-differential operators and the Nash-Moser theorem, volume 82 of Graduate Studies in Mathematics. American Mathematical Society, Providence, RI, 2007. Translated from the 1991 French original by Stephen S. Wilson.

[2] X. Antoine and C. Besse. Construction, structure and asymptotic approximations of a microdifferential transparent boundary condition for the linear Schrödinger equation. Journal de mathématiques pures et appliquées, 80(7):701-738, 2001.

[3] X. Antoine, C. Besse, and P. Klein. Absorbing boundary conditions for the onedimensional Schrödinger equation with an exterior repulsive potential. Journal of Computational Physics, 228(2):312-335, 2009.

[4] X. Antoine and E. Lorin. An analysis of Schwarz waveform relaxation domain decomposition methods for the imaginary-time linear Schrödinger and Gross-Pitaevskii equations, 2015.

[5] X. Antoine, E. Lorin, and A. Bandrauk. Domain decomposition method and highorder absorbing boundary conditions for the numerical simulation of the time dependent Schrödinger equation with ionization and recombination by intense electric field. Journal of Scientific Computing, 64(3):620-646, 2015.

[6] V. Dolean, P. Jolivet, and F. Nataf. An Introduction to Domain Decomposition Methods: Algorithms, Theory, and Parallel Implementation, volume 144. SIAM, 2015.

[7] L. C. Evans. Partial differential equations. Graduate Studies in Mathematics, 19, 1998. 
[8] M. Gander and L. Halpern. Optimized Schwarz waveform relaxation methods for advection reaction diffusion problems. SIAM Journal on Numerical Analysis, 45(2):666-697, 2007.

[9] L. Halpern and J. Szeftel. Optimized and quasi-optimal Schwarz waveform relaxation for the one-dimensional Schrödinger equation. Mathematical Models and Methods in Applied Sciences, 20(12):2167-2199, 2010.

[10] L. Hörmander. Linear partial differential operators, volume 116. Springer, 2013.

[11] R. Lascar. Propagation des singularités des solutions d'équations pseudo-différentielles quasi-homogènes. In Annales de l'institut Fourier, volume 27, pages 79-123, 1977.

[12] J-L. Lions and E. Magenes. Problemes aux limites non homogenes et applications. vol. 1. 1968.

[13] R.C. McOwen. Partial differential equations: Methods and applications. Prentice Hall, 1996.

[14] W. Rudin. Principles of mathematical analysis, volume 3. McGraw-Hill New York, 1964. 